ESAIM: COCV

DOI: $10.1051 / \mathrm{cocv} / 2015048$
ESAIM: Control, Optimisation and Calculus of Variations

www.esaim-cocv.org

\title{
ANALYSIS OF SPATIO-TEMPORALLY SPARSE OPTIMAL CONTROL PROBLEMS OF SEMILINEAR PARABOLIC EQUATIONS*
}

\author{
Eduardo Casas $^{1}$, Roland Herzog ${ }^{2}$ and Gerd Wachsmuth ${ }^{2}$
}

\begin{abstract}
Optimal control problems with semilinear parabolic state equations are considered. The objective features one out of three different terms promoting various spatio-temporal sparsity patterns of the control variable. For each problem, first-order necessary optimality conditions, as well as secondorder necessary and sufficient optimality conditions are proved. The analysis includes the case in which the objective does not contain the squared norm of the control.
\end{abstract}

Mathematics Subject Classification. 49K20, 49J52, 35K58, 65K10.

Received March 20, 2015. Revised June 24, 2015. Accepted September 10, 2015.

\section{INTRODUCTION}

In this paper, we analyze some optimal control problems governed by semilinear parabolic equations where the cost functional involves a functional $j$ acting on the control which promotes the sparsity of the optimal control. We present three different choices for the functional $j$. Each of these choices induces a different spatio-temporal sparsity pattern for the optimal control, all of them being interesting. The control problems are formulated as follows

$$
\left(\mathrm{P}_{\nu}\right)\left\{\begin{array}{l}
\text { Minimize } J_{\nu}(u)=F_{\nu}(u)+\mu j(u) \\
\alpha \leq u(x, t) \leq \beta \quad \text { for a.a. }(x, t) \in \Omega_{T}=\Omega \times(0, T)
\end{array}\right.
$$

where

$$
F_{\nu}(u)=\int_{\Omega_{T}} L\left(x, t, y_{u}(x, t)\right) \mathrm{d} x \mathrm{~d} t+\frac{\nu}{2} \int_{\Omega_{T}} u^{2}(x, t) \mathrm{d} x \mathrm{~d} t
$$

Keywords and phrases. Optimal control, directional sparsity, second-order optimality conditions, semilinear parabolic equations.

* The first author was partially supported by Spanish Ministerio de Economía y Competitividad under the project MTM201122711.

1 Departamento de Matemática Aplicada y Ciencias de la Computación, E.T.S.I. Industriales y de Telecomunicación, Universidad de Cantabria, Av. Los Castros s/n, 39005 Santander, Spain. eduardo.casas@unican.es

2 Technische Universität Chemnitz, Faculty of Mathematics, Professorship Numerical Methods (Partial Differential Equations), 09107 Chemnitz, Germany. roland.herzog@mathematik.tu-chemnitz.de; gerd.wachsmuth@mathematik.tu-chemnitz.de 
$j: L^{2}\left(\Omega_{T}\right) \rightarrow \mathbb{R}$ is a Lipschitz continuous and convex but not Fréchet differentiable function, $\nu \geq 0, \mu>0$. The state $y_{u}$ is the solution of the semilinear, parabolic equation

$$
\left\{\begin{array}{l}
\partial_{t} y_{u}+A y_{u}+a\left(x, t, y_{u}\right)=u \text { in } \Omega_{T} \\
y_{u}=0 \text { on } \Sigma_{T}=\Gamma \times(0, T), \\
y_{u}(0)=y_{0} \text { in } \Omega
\end{array}\right.
$$

Here, $A$ is the linear elliptic operator

$$
A y=-\sum_{i, j=1}^{n} \partial_{x_{j}}\left[a_{i j}(x) \partial_{x_{i}} y\right] .
$$

We mention that it is possible to replace the Dirichlet boundary condition $y_{u}=0$ by a Neumann boundary condition $\partial_{n_{A}} y_{u}=g$ with $g \in L^{p}(\Sigma)$, provided that $p$ is sufficiently large, so that $L^{\infty}$ estimates for the solution of the boundary value problem are obtained.

The goal of this paper is to carry out the first and the second-order analysis of $\left(\mathrm{P}_{\nu}\right)$. This analysis will be done for each of the three following choices for the functional $j$

$$
\begin{aligned}
& j_{1}: L^{1}\left(\Omega_{T}\right) \rightarrow \mathbb{R}, \quad j_{1}(u)=\|u\|_{L^{1}\left(\Omega_{T}\right)}=\int_{\Omega_{T}}|u(x, t)| \mathrm{d} x \mathrm{~d} t \\
& j_{2}: L^{2}\left(0, T ; L^{1}(\Omega)\right) \rightarrow \mathbb{R}, \quad j_{2}(u)=\|u\|_{L^{2}\left(0, T ; L^{1}(\Omega)\right)}=\left[\int_{0}^{T}\|u(t)\|_{L^{1}(\Omega)}^{2} \mathrm{~d} t\right]^{1 / 2}, \\
& j_{3}: L^{1}\left(\Omega ; L^{2}(0, T)\right) \rightarrow \mathbb{R}, \quad j_{3}(u)=\|u\|_{L^{1}\left(\Omega ; L^{2}(0, T)\right)}=\int_{\Omega}\|u(x)\|_{L^{2}(0, T)} \mathrm{d} x .
\end{aligned}
$$

When we take $j=j_{1}$, the corresponding problem $\left(\mathrm{P}_{\nu}\right)$ will be denoted by $\left(\mathrm{P}_{\nu}^{1}\right)$. Analogously, we define the the control problems $\left(\mathrm{P}_{\nu}^{2}\right)$ and $\left(\mathrm{P}_{\nu}^{3}\right)$ corresponding to the other two functionals $j_{2}$ and $j_{3}$.

Problems with the functional $j_{1}$ and linear elliptic equations were first analyzed in [15]. Later on, a secondorder analysis in the presence of semilinear elliptic state equations was provided in [5] and adapted to measurevalued controls in [4]. Note that the functional $j_{1}$ does not provide control over the structure of the spatiotemporal sparsity pattern of the optimal control.

Problems involving the functional $j_{2}$ have been studied in [6], again with measure-valued controls in place of $L^{1}$. The term $j_{2}$ promotes optimal controls which are spatially sparse, for almost all points in time. The spatial sparsity pattern may change over time.

Finally, the functional $j_{3}$ promotes sparsity patterns which are spatially sparse and constant throughout the time interval. Corresponding optimal control problems with linear elliptic and parabolic equations have been studied in [11], and the term directionally sparse controls was coined there. An extension of this work to measure-valued controls can be found in [13].

The motivation for considering measure-valued controls in some of the above references is that problem $\left(\mathrm{P}_{\nu}\right)$ is not well-posed in $L^{2}\left(\Omega_{T}\right)$ in case $\nu=0$, provided that control constraints are also absent (i.e. $\alpha=-\infty$, $\beta=\infty)$. In this situation, minimizing sequences will converge in the weak- $*$ topology of an appropriate measure space. Due to the presence of the control bounds in $\left(\mathrm{P}_{\nu}\right)$, we can obtain solutions in $L^{2}\left(\Omega_{T}\right)$ even when $\nu=0$, see Theorem 2.4 below.

Unless stated otherwise, the references above pertain to problems with linear state equations and convex objectives, hence no second-order analysis is necessary.

Besides the first-order necessary conditions, we derive in this paper second-order necessary and sufficient conditions for the non-convex problems $\left(\mathrm{P}_{\nu}^{1}\right)-\left(\mathrm{P}_{\nu}^{3}\right)$, which, in case $\nu>0$, both use the same cone of critical directions and thus provide the minimal gap between second-order necessary and sufficient conditions. Note that the second-order directional derivatives $v \mapsto j^{\prime \prime}\left(u ; v^{2}\right)$ of the above functionals do not exist in all directions. 
It is therefore necessary to define suitable substitutes, see (4.5)-(4.7). It was already shown in [5] that $j_{1}^{\prime \prime}=0$ can be used in case of the first functional. This is however not true for $j_{2}$ and $j_{3}$.

The paper is organized as follows. We summarize our assumptions and some preliminary results in the following section. Section 3 is devoted to the derivation of first-order optimality conditions. As a corollary, we analyze the sparsity structure of the solution in all three cases, see Remark 3.11 and Figure 1. In Section 4 we address second-order necessary optimality conditions and in Section 5 the second-order sufficient conditions.

We point out that the case $\nu=0$ is explicitly included in the analysis. The only problem that remains open is the second-order sufficient condition for problem $\left(\mathrm{P}_{0}^{3}\right)$. We comment on this case at the end of Section 5 .

\section{Assumptions AND PRELiminary RESults}

Throughout the paper, $\Omega$ denotes an open, bounded subset of $\mathbb{R}^{n}, 1 \leq n \leq 3$, with a Lipschitz boundary $\Gamma$; see ([14], Sect. 1.3). The final time $T>0$ is given and fixed. We make the following assumptions on the functions and parameters involved in the control problem $\left(\mathrm{P}_{\nu}\right)$.

Assumption 1. The coefficients of $A$ have the following regularity properties: $a_{i j} \in L^{\infty}(\Omega)$ and

$$
\exists \Lambda>0 \text { such that } \sum_{i, j=1}^{n} a_{i j}(x) \xi_{i} \xi_{j} \geq \Lambda|\xi|^{2} \text { for a.a. } x \in \Omega \text { and } \forall \xi \in \mathbb{R}^{n} .
$$

Assumption 2. The initial datum $y_{0} \in L^{\infty}(\Omega)$ and $a: \Omega_{T} \times \mathbb{R} \longrightarrow \mathbb{R}$ is a Carathéodory function of class $C^{2}$ with respect to the last variable, satisfying the following assumptions

$$
\begin{aligned}
& \left\{\begin{array}{l}
\exists \psi_{a} \in L^{\hat{p}}\left(0, T ; L^{\hat{q}}(\Omega)\right) \text { and } C_{a} \in \mathbb{R} \text { such that } \\
a(x, t, y) y \geq \psi_{a}(x, t)+C_{a} y^{2} \text { for a.a. }(x, t) \in \Omega_{T} \text { and } \forall y \in \mathbb{R},
\end{array}\right. \\
& \left\{\begin{array}{l}
a(\cdot, \cdot, 0) \in L^{\hat{p}}\left(0, T ; L^{\hat{q}}(\Omega)\right) \text { and } \forall M>0 \exists C_{M}>0 \text { such that } \\
\left|\frac{\partial^{j} a}{\partial y^{j}}(x, t, y)\right| \leq C_{M} \text { for a.a. }(x, t) \in \Omega_{T}, \forall|y| \leq M, \text { with } j=1,2
\end{array}\right. \\
& \left\{\begin{array}{l}
\forall \rho>0 \text { and } \forall M>0 \exists \varepsilon_{M, \rho}>0 \text { such that for a.a. }(x, t) \in \Omega_{T} \\
\left|\frac{\partial^{2} a}{\partial y^{2}}\left(x, t, y_{2}\right)-\frac{\partial^{2} a}{\partial y^{2}}\left(x, t, y_{1}\right)\right| \leq \rho, \forall\left|y_{i}\right| \leq M, \text { and }\left|y_{2}-y_{1}\right| \leq \varepsilon_{M, \rho},
\end{array}\right.
\end{aligned}
$$

where $\hat{p}, \hat{q} \in[2,+\infty]$, with $\frac{1}{\hat{p}}+\frac{n}{2 \hat{q}}<1$.

Assumption 3. We also assume $-\infty<\alpha<0<\beta<+\infty, \mu>0, \nu \geq 0$, and $L: \Omega_{T} \times \mathbb{R} \longrightarrow \mathbb{R}$ is a Carathéodory function of class $C^{2}$ with respect to the last variable such that $L(\cdot, \cdot, 0) \in L^{1}\left(\Omega_{T}\right)$. Furthermore, for every $M>0$ there exists a function $\psi_{M} \in L^{\hat{p}}\left(0, T ; L^{\hat{q}}(\Omega)\right)$ satisfying

$$
\begin{aligned}
& \left\{\begin{array}{l}
\forall M>0 \exists \psi_{M} \in L^{\hat{p}}\left(0, T ; L^{\hat{q}}(\Omega)\right) \text { and } C_{M}>0 \text { such that } \\
\left|\frac{\partial L}{\partial y}(x, t, y)\right| \leq \psi_{M}(x, t) \forall|y| \leq M \text { and for a.a. }(x, t) \in \Omega_{T}, \\
\left|\frac{\partial^{2} L}{\partial y^{2}}(x, t, y)\right| \leq C_{M} \forall|y| \leq M \text { and for a.a. }(x, t) \in \Omega_{T},
\end{array}\right. \\
& \left\{\begin{array}{l}
\forall \rho>0 \text { and } \forall M>0 \exists \varepsilon_{M, \rho}>0 \text { such that for a.a. }(x, t) \in \Omega_{T} \\
\left|\frac{\partial^{2} L}{\partial y^{2}}\left(x, t, y_{2}\right)-\frac{\partial^{2} L}{\partial y^{2}}\left(x, t, y_{1}\right)\right| \leq \rho, \forall\left|y_{i}\right| \leq M, \text { and }\left|y_{2}-y_{1}\right| \leq \varepsilon_{M, \rho} .
\end{array}\right.
\end{aligned}
$$

In the sequel, we will denote the set of feasible controls by

$$
\mathbb{K}=\left\{u \in L^{\infty}\left(\Omega_{T}\right): \alpha \leq u(x, t) \leq \beta \text { for a.a. }(x, t) \in \Omega_{T}\right\} .
$$


As usual, we denote by $W(0, T)$ the space of functions $y \in L^{2}\left(0, T ; H_{0}^{1}(\Omega)\right)$ such that $\partial_{t} y \in L^{2}\left(0, T ; H^{-1}(\Omega)\right)$. It is well-known that every function $y \in W(0, T)$ belongs, after a modification on a set of zero Lebesgue measure, to $C\left([0, T], L^{2}(\Omega)\right)$. Now, we analyze the existence, uniqueness and regularity of a solution of (1.1).

Theorem 2.1. Under the Assumptions 1 and 2 , for all $u \in L^{\hat{p}}\left(0, T ; L^{\hat{q}}(\Omega)\right)$ equation (1.1) has a unique solution $y_{u} \in W(0, T) \cap L^{\infty}\left(\Omega_{T}\right)$. Moreover, the mapping $G: L^{\hat{p}}\left(0, T ; L^{\hat{q}}(\Omega)\right) \longrightarrow W(0, T) \cap L^{\infty}\left(\Omega_{T}\right)$, defined by $G(u)=y_{u}$, is of class $C^{2}$. For all elements $u, v, v_{1}$ and $v_{2}$ of $L^{\hat{p}}\left(0, T ; L^{\hat{q}}(\Omega)\right)$, the functions $z_{v}=G^{\prime}(u) v$ and $z_{v_{1} v_{2}}=G^{\prime \prime}(u)\left(v_{1}, v_{2}\right)$ are the solutions of the problems

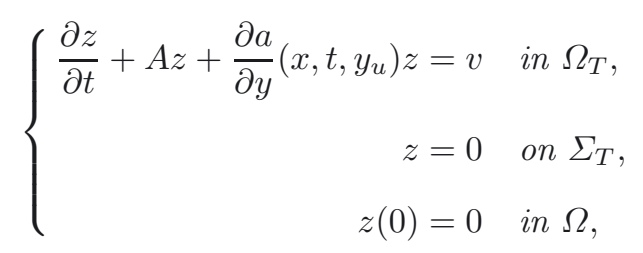

and

$$
\left\{\begin{aligned}
\frac{\partial z}{\partial t}+A z+\frac{\partial a}{\partial y}\left(x, t, y_{u}\right) z+\frac{\partial^{2} a}{\partial y^{2}}\left(x, t, y_{u}\right) z_{v_{1}} z_{v_{2}}=0 & \text { in } \Omega_{T} \\
z=0 & \text { on } \Sigma_{T} \\
z(0)=0 & \text { in } \Omega,
\end{aligned}\right.
$$

respectively.

The proof of the existence and uniqueness of a solution of $(1.1)$ in $W(0, T) \cap L^{\infty}\left(\Omega_{T}\right)$ is standard. The reader is referred, for instance, to [2] where the arguments used for a Robin boundary condition can be easily adapted to the Dirichlet case. For the proof of the differentiability we can proceed as follows. We set

$$
Y=\left\{y \in W(0, T) \cap L^{\infty}\left(\Omega_{T}\right): \frac{\partial y}{\partial t}+A y \in L^{\hat{p}}\left(0, T ; L^{\hat{q}}(\Omega)\right)\right\}
$$

endowed with the graph norm. $Y$ is a Banach space and $Y \subset C\left([0, T], L^{2}(\Omega)\right)$. Indeed, recall that $L^{\hat{p}}\left(0, T ; L^{\hat{q}}(\Omega)\right) \subset L^{2}\left(\Omega_{T}\right) \subset L^{2}\left(0, T ; H^{-1}(\Omega)\right)$. Then, we have $y(0)=\lim _{t \rightarrow 0} y(t) \in L^{\infty}(\Omega)$. Now, we define

$$
\begin{gathered}
\mathcal{F}: Y \times L^{\hat{p}}\left(0, T ; L^{\hat{q}}(\Omega)\right) \longrightarrow L^{\hat{p}}\left(0, T ; L^{\hat{q}}(\Omega)\right) \times L^{\infty}\left(\Omega_{T}\right), \\
\mathcal{F}(y, u)=\left(\frac{\partial y}{\partial t}+A y+a(x, t, y)-u, y(0)-y_{0}\right) .
\end{gathered}
$$

Using that $y \in L^{\infty}\left(\Omega_{T}\right)$ and $(2.3)$ we deduce that $a(\cdot, \cdot, y) \in L^{\hat{p}}\left(0, T ; L^{\hat{q}}(\Omega)\right)$. Hence, $\mathcal{F}$ is well defined and we can apply the implicit function theorem to deduce that $G$ is of class $C^{2}$ and to show that (2.7) and (2.8) represent its first and second derivatives, respectively.

Remark 2.2. In Assumptions 2 and 3, the condition $\hat{p}, \hat{q} \geq 2$ is not necessary for Theorem 2.1. Indeed, it is enough to impose $\hat{p}, \hat{q} \in[1,+\infty]$. However, the assumption $\hat{p}, \hat{q} \in[2,+\infty]$ is useful to get some extra regularity for $y_{u}$ and it will simplify our presentation, avoiding some technicalities.

Now, we have the following differentiability result. 
Theorem 2.3. Under the Assumptions $1-3, F_{\nu}: L^{\hat{p}}\left(0, T ; L^{\hat{q}}(\Omega)\right) \longrightarrow \mathbb{R}$ is of class $C^{2}$. Moreover, for all $u, v, v_{1}$ and $v_{2}$ of $L^{\hat{p}}\left(0, T ; L^{\hat{q}}(\Omega)\right)$ we have

$$
\begin{gathered}
F_{\nu}^{\prime}(u) v=\int_{\Omega_{T}}\left(\frac{\partial L}{\partial y}\left(x, t, y_{u}\right) z_{v}+\nu u v\right) \mathrm{d} x \mathrm{~d} t=\int_{\Omega_{T}}\left(\varphi_{u}+\nu u\right) v \mathrm{~d} x \mathrm{~d} t \\
F_{\nu}^{\prime \prime}(u)\left(v_{1}, v_{2}\right)=\int_{\Omega_{T}}\left\{\left(\frac{\partial^{2} L}{\partial y^{2}}\left(x, t, y_{u}\right)-\varphi_{u} \frac{\partial^{2} a}{\partial y^{2}}\left(x, t, y_{u}\right)\right) z_{v_{1}} z_{v_{2}}+\nu v_{1} v_{2}\right\} \mathrm{d} x \mathrm{~d} t,
\end{gathered}
$$

where $z_{v_{i}}=G^{\prime}(u) v_{i}, i=1,2$, and $\varphi_{u} \in W(0, T) \cap L^{\infty}\left(\Omega_{T}\right)$ is the solution of

$$
\left\{\begin{aligned}
-\frac{\partial \varphi}{\partial t}+A^{*} \varphi+\frac{\partial a}{\partial y}\left(x, t, y_{u}\right) \varphi & =\frac{\partial L}{\partial y}\left(x, t, y_{u}\right) & & \text { in } \Omega_{T}, \\
\varphi & =0 & & \text { on } \Sigma_{T}, \\
\varphi(T) & =0 & & \text { in } \Omega,
\end{aligned}\right.
$$

$A^{*}$ being the adjoint operator of $A$.

The fact that $F_{\nu}$ is of class $C^{2}$ is an immediate consequence of Theorem 2.1 and the chain rule. On the other hand, since $y_{u} \in L^{\infty}\left(\Omega_{T}\right)$, we deduce from $(2.5)$ that $\frac{\partial L}{\partial y}\left(\cdot, \cdot, y_{u}\right) \in L^{\hat{p}}\left(0, T ; L^{\hat{q}}(\Omega)\right)$, which implies that $\varphi_{u}$ is well defined and enjoys the indicated regularity. The formulas (2.9) and (2.10) follow from standard computations.

Analogously to $Y$, we define the space

$$
\Phi=\left\{\varphi \in W(0, T) \cap L^{\infty}\left(\Omega_{T}\right):-\frac{\partial \varphi}{\partial t}+A^{*} y \in L^{\hat{p}}\left(0, T ; L^{\hat{q}}(\Omega)\right)\right\},
$$

endowed with the graph norm. As established for $Y$, we also have the embedding $\Phi \subset C\left([0, T], L^{2}(\Omega)\right)$.

We conclude this section by stating the following theorem, whose proof follows from classical arguments by taking a minimizing sequence.

Theorem 2.4. Problem $\left(\mathrm{P}_{\nu}\right)$ has at least one solution $\bar{u}_{\nu}$.

\section{First-ORDER OPTIMALITy CONDITIONS}

Since $\left(\mathrm{P}_{\nu}\right)$ is not a convex problem, we will deal with local solutions. We say that $\bar{u}_{\nu}$ is a local solution of $\left(\mathrm{P}_{\nu}\right)$ if there exists $\varepsilon>0$ such that $J_{\nu}\left(\bar{u}_{\nu}\right) \leq J_{\nu}(u)$ for all $u \in B_{\varepsilon}\left(\bar{u}_{\nu}\right)$, where $B_{\varepsilon}\left(\bar{u}_{\nu}\right)$ denotes the open ball in $L^{2}\left(\Omega_{T}\right)$ centered at $\bar{u}_{\nu}$ and with radius $\varepsilon$. Moreover, $\bar{u}_{\nu}$ is said a strict local minimum if the previous inequality is strict for every $u \in B_{\varepsilon}\left(\bar{u}_{\nu}\right)$ different from $\bar{u}_{\nu}$.

The next theorem states the first-order optimality conditions satisfied by a local minimum of $\left(\mathrm{P}_{\nu}\right)$. To this end, we recall that the tangent cone $\mathcal{T}_{\mathbb{K}}\left(\bar{u}_{\nu}\right)$ of $\mathbb{K}$ at $\bar{u}_{\nu}$ with respect to the $L^{2}\left(\Omega_{T}\right)$-topology is given by

$$
\mathcal{T}_{\mathbb{K}}\left(\bar{u}_{\nu}\right)=\left\{v \in L^{2}\left(\Omega_{T}\right): v(x, t) \geq 0 \text { if } \bar{u}_{\nu}(x, t)=\alpha \text { and } v(x, t) \leq 0 \text { if } \bar{u}_{\nu}(x, t)=\beta\right\},
$$

see for instance ([1], Lem. 6.34).

Theorem 3.1. If $\bar{u}_{\nu}$ is a local minimum of $\left(\mathrm{P}_{\nu}\right)$, then there exist $\bar{y}_{\nu} \in Y, \bar{\varphi}_{\nu} \in \Phi$, and $\bar{\lambda}_{\nu} \in \partial j\left(\bar{u}_{\nu}\right)$ such that

$$
\begin{aligned}
& \left\{\begin{aligned}
\partial_{t} \bar{y}_{\nu}+A \bar{y}_{\nu}+a\left(x, t, \bar{y}_{\nu}\right)=\bar{u}_{\nu} & \text { in } \Omega_{T}, \\
\bar{y}_{\nu}=0 & \text { on } \Sigma_{T}, \\
\bar{y}_{\nu}(0)=y_{0} & \text { in } \Omega,
\end{aligned}\right. \\
& \left\{\begin{aligned}
-\partial_{t} \bar{\varphi}_{\nu}+A^{*} \bar{\varphi}_{\nu}+\frac{\partial a}{\partial y}\left(x, t, \bar{y}_{\nu}\right) \bar{\varphi}_{\nu}=\frac{\partial L}{\partial y}\left(x, t, \bar{y}_{\nu}\right) & \text { in } \Omega_{T}, \\
\bar{\varphi}_{\nu}=0 & \text { on } \Sigma_{T}, \\
\bar{\varphi}_{\nu}(T)=0 & \text { in } \Omega,
\end{aligned}\right. \\
& \int_{\Omega_{T}}\left(\bar{\varphi}_{\nu}+\nu \bar{u}_{\nu}+\mu \bar{\lambda}_{\nu}\right) v \mathrm{~d} x \mathrm{~d} t \geq 0 \quad \forall v \in \mathcal{T}_{\mathbb{K}}\left(\bar{u}_{\nu}\right) .
\end{aligned}
$$


In the theorem, $\partial j\left(\bar{u}_{\nu}\right)$ denotes the subdifferential in the sense of convex analysis of $j$ at the point $\bar{u}_{\nu}$.

Proof. Since $j$ is convex and Lipschitz, we have for every $u \in \mathbb{K}$

$$
F_{\nu}^{\prime}\left(\bar{u}_{\nu}\right)\left(u-\bar{u}_{\nu}\right)+\mu j(u)-\mu j\left(\bar{u}_{\nu}\right) \geq \lim _{\rho \searrow 0} \frac{J_{\nu}\left(\bar{u}_{\nu}+\rho\left(u-\bar{u}_{\nu}\right)\right)-J_{\nu}\left(\bar{u}_{\nu}\right)}{\rho} \geq 0 .
$$

Therefore, $\bar{u}_{\nu}$ is a solution of the convex optimization problem

$$
\min _{u \in L^{\infty}\left(\Omega_{T}\right)} I_{\nu}(u)=F_{\nu}^{\prime}\left(\bar{u}_{\nu}\right) u+\mu j(u)+I_{\mathbb{K}}(u),
$$

where $I_{\mathbb{K}}$ denotes the indicator function of the convex set $\mathbb{K}$. Since both, $F_{\nu}^{\prime}\left(\bar{u}_{\nu}\right)$ and $j$ are continuous, we can apply the Moreau-Rockafellar Theorem (see [9], Chap. I, Prop. 5.6), and obtain

$$
0 \in \partial I_{\nu}\left(\bar{u}_{\nu}\right)=F_{\nu}^{\prime}\left(\bar{u}_{\nu}\right)+\mu \partial j\left(\bar{u}_{\nu}\right)+\partial I_{\mathbb{K}}\left(\bar{u}_{\nu}\right) .
$$

Therefore, there exists $\bar{\lambda}_{\nu} \in \partial j\left(\bar{u}_{\nu}\right)$ such that $-\left[F_{\nu}^{\prime}\left(\bar{u}_{\nu}\right)+\mu \bar{\lambda}_{\nu}\right] \in \partial I_{\mathbb{K}}\left(\bar{u}_{\nu}\right)$. Finally, from this relation and taking $\bar{y}_{\nu}$ and $\bar{\varphi}_{\nu}$ solutions of (3.2) and (3.3), respectively, we deduce (3.4) with (2.9), (3.1) and the fact that $\bar{\varphi}_{\nu}+\nu \bar{u}_{\nu}+\mu \bar{\lambda}_{\nu} \in L^{2}\left(\Omega_{T}\right)$.

Now, we use the optimality system (3.2)-(3.4) to deduce the sparse structure of $\bar{u}_{\nu}$ for the three choices of $j$.

\subsection{Problem $\left(\mathrm{P}_{\nu}^{1}\right)$}

Recall the functional $j_{1}: L^{1}\left(\Omega_{T}\right) \longrightarrow \mathbb{R}$ defined by

$$
j_{1}(u)=\int_{\Omega_{T}}|u(x, t)| \mathrm{d} x \mathrm{~d} t .
$$

Let us state some properties of $j_{1}$. First, a simple computation shows that $\lambda \in \partial j_{1}(u)$ if and only if $\lambda \in L^{\infty}\left(\Omega_{T}\right)$ and

$$
\begin{cases}\lambda(x, t)=+1 & \text { if } u(x, t)>0, \\ \lambda(x, t)=-1 & \text { if } u(x, t)<0, \\ \lambda(x, t) \in[-1,+1] & \text { if } u(x, t)=0,\end{cases}
$$

holds a.e. in $\Omega_{T}$. Moreover, the directional derivatives of $j_{1}$ are given by

$$
j_{1}^{\prime}(u ; v)=\lim _{\rho \searrow 0} \frac{j_{1}(u+\rho v)-j_{1}(u)}{\rho}=\int_{\Omega_{T, u}^{+}} v(x, t) \mathrm{d} t \mathrm{~d} x-\int_{\Omega_{T, u}^{-}} v(x, t) \mathrm{d} t \mathrm{~d} x+\int_{\Omega_{T, u}^{0}}|v(x, t)| \mathrm{d} t \mathrm{~d} x,
$$

where $\Omega_{T, u}^{+}, \Omega_{T, u}^{-}$and $\Omega_{T, u}^{0}$ denote the set of points of $\Omega_{T}$ where $u$ is positive, negative or zero, respectively.

Now, taking $j=j_{1}$ in Theorem 3.1, we deduce from the variational inequality (3.4) the following properties.

Corollary 3.2. Let $\bar{u}_{\nu}, \bar{\varphi}_{\nu}$ and $\bar{\lambda}_{\nu}$ be as in Theorem 3.1, then the following relations hold for almost all $(x, t) \in \Omega_{T}$

$$
\begin{gathered}
\text { if } \nu>0 \text { then }\left\{\begin{array}{l}
\bar{u}_{\nu}(x, t)=0 \Leftrightarrow\left|\bar{\varphi}_{\nu}(x, t)\right| \leq \mu, \\
\bar{u}_{\nu}(x, t)=\operatorname{Proj}_{[\alpha, \beta]}\left(-\frac{1}{\nu}\left[\bar{\varphi}_{\nu}(x, t)+\mu \bar{\lambda}_{\nu}(x, t)\right]\right),
\end{array}\right. \\
\text { if } \nu=0 \text { then }\left\{\begin{array}{l}
\left|\bar{\varphi}_{\nu}(x, t)\right|<\mu \Rightarrow \bar{u}_{\nu}(x, t)=0, \\
\bar{\varphi}_{\nu}(x, t)>\mu \Rightarrow \bar{u}_{\nu}(x, t)=\alpha, \\
\bar{\varphi}_{\nu}(x, t)<-\mu \Rightarrow \bar{u}_{\nu}(x, t)=\beta,
\end{array}\right. \\
\bar{\lambda}_{\nu}(x, t)=\operatorname{Proj}_{[-1,+1]}\left(-\frac{1}{\mu} \bar{\varphi}_{\nu}(x, t)\right) .
\end{gathered}
$$


Here, $\operatorname{Proj}_{[a, b]}(c)$ refers to the projection of $c \in \mathbb{R}$ onto the interval $[a, b] \subset \mathbb{R}$. Moreover, $\bar{\lambda}_{\nu} \in L^{2}\left(0, T ; H_{0}^{1}(\Omega)\right)$ holds and it is unique. Finally, if $\nu>0$, we also have that $\bar{u}_{\nu} \in L^{2}\left(0, T ; H_{0}^{1}(\Omega)\right)$.

The proofs of (3.7) and (3.9) were given in ([5], Cor.3.2) and (3.8) can be found in ([3], Thm. 3.1). In both cases, the control problems are elliptic, but there is no change in the proofs (up to the replacement of the argument $x$ by $(x, t)$ ) with the parabolic case, since the above corollary is just a consequence of (3.4). In the case $\nu>0$, the first equivalence of (3.7) shows the sparsity of $\bar{u}_{\nu}$ and the regularity follows from the second relation. For $\nu=0$, if the set of points $(x, t) \in \Omega_{T}$ where $\left|\bar{\varphi}_{\nu}(x, t)\right|=\mu$ has a zero Lebesgue measure (which is expected in many cases), then $\bar{u}_{\nu}(x, t) \in\{\alpha, 0, \beta\}$ for almost all $(x, t) \in \Omega_{T}$, which means that the optimal control has a bang-bang-bang structure.

Remark 3.3. From the state equation (3.2) we can get an estimate $\left\|\bar{y}_{\nu}\right\|_{L^{\infty}\left(\Omega_{T}\right)} \leq M$, with $M$ depending on $\alpha$ and $\beta$, but independent of $\bar{u}_{\nu}$. Using (2.5), we obtain from (3.3) a similar estimate $\left\|\bar{\varphi}_{\nu}\right\|_{L^{\infty}\left(\Omega_{T}\right)} \leq M^{\prime}$, with $M^{\prime}$ independent of $\bar{u}_{\nu}$. Hence, from (3.7) and (3.8) we conclude that $\bar{u}_{\nu} \equiv 0$ if $\mu>M^{\prime}$. Therefore, we may influence the size of an optimal control's support by adjusting $\mu$ in the interval $\left[0, M^{\prime}\right]$.

\subsection{Problem $\left(\mathrm{P}_{\nu}^{2}\right)$}

We recall that $j_{2}: L^{2}\left(0, T ; L^{1}(\Omega)\right) \longrightarrow \mathbb{R}$ is defined by

$$
j_{2}(u)=\left(\int_{0}^{T}\|u(t)\|_{L^{1}(\Omega)}^{2} \mathrm{~d} t\right)^{1 / 2}=\left[\int_{0}^{T}\left(\int_{\Omega}|u(x, t)| \mathrm{d} x\right)^{2} \mathrm{~d} t\right]^{1 / 2} .
$$

Hereafter, $\|\cdot\|_{L^{2}\left(L^{1}\right)}$ will denote this norm of the space $L^{2}\left(0, T ; L^{1}(\Omega)\right)$. For $u \in L^{2}\left(\Omega_{T}\right)$, we define

$$
\|u\|_{L^{2}\left(L^{\infty}\right)}^{2}=\|u\|_{L^{2}\left(0, T ; L^{\infty}(\Omega)\right)}^{2}=\int_{0}^{T} \operatorname{ess~sup}_{x \in \Omega}|u(x, t)|^{2} \mathrm{~d} t \in[0,+\infty]
$$

and

$$
L^{2}\left(0, T ; L^{\infty}(\Omega)\right)=\left\{u \in L^{2}\left(\Omega_{T}\right):\|u\|_{L^{2}\left(L^{\infty}\right)}<\infty\right\} .
$$

Note that $L^{2}\left(0, T ; L^{\infty}(\Omega)\right)$ is not a Bochner-Lebesgue space, since its elements are only weakly-* measurable w.r.t. $L^{\infty}(\Omega)$. From Theorem 8.20 .3 of [8], we have that $L^{2}\left(0, T ; L^{\infty}(\Omega)\right)$ is the dual space of $L^{2}\left(0, T ; L^{1}(\Omega)\right)$.

Now, we study the properties of the elements of $\partial j_{2}(u)$.

Proposition 3.4. For $u \neq 0, \lambda \in \partial j_{2}(u)$ is equivalent to $\lambda \in L^{2}\left(0, T ; L^{\infty}(\Omega)\right)$ and

$$
\lambda(x, t) \in \operatorname{Sign}(u(x, t)) \frac{\|u(t)\|_{L^{1}(\Omega)}}{\|u\|_{L^{2}\left(L^{1}\right)}} \quad \text { a.e. in } \Omega_{T},
$$

where

$$
\operatorname{Sign}(\theta)=\left\{\begin{array}{cc}
+1 & \text { if } \theta>0 \\
-1 & \text { if } \theta<0 \\
{[-1,+1]} & \text { if } \theta=0
\end{array}\right.
$$

In case $u=0$, we have $\partial j_{2}(u)=\left\{\lambda \in L^{2}\left(0, T ; L^{\infty}(\Omega)\right):\|\lambda\|_{L^{2}\left(L^{\infty}\right)} \leq 1\right\}$. Moreover, for $\lambda \in \partial j_{2}(u)$ we have

$$
\left\{\begin{array}{l}
\operatorname{supp} u^{+}(t) \subset\left\{x \in \Omega: \lambda(x, t)=+\|\lambda(t)\|_{L^{\infty}(\Omega)}\right\} \\
\operatorname{supp} u^{-}(t) \subset\left\{x \in \Omega: \lambda(x, t)=-\|\lambda(t)\|_{L^{\infty}(\Omega)}\right.
\end{array}\right\} \text { a.e. in }(0, T) .
$$


Proof. Since $j_{2}$ is the norm of $L^{2}\left(0, T ; L^{1}(\Omega)\right)$, we have the characterization

$$
\partial j_{2}(u)=\left\{\lambda \in L^{2}\left(0, T ; L^{\infty}(\Omega)\right):\|\lambda\|_{L^{2}\left(L^{\infty}\right)} \leq 1 \text { and } \int_{\Omega_{T}} \lambda u \mathrm{~d} x \mathrm{~d} t=\|u\|_{L^{2}\left(L^{1}\right)}\right\},
$$

see, e.g., ([12], p. 56, [10], Lem. 2.1).

Using this characterization, it is easy to check that (3.10) implies $\lambda \in \partial j_{2}(u)$.

Now, we prove that (3.10) holds for $\lambda \in \partial j_{2}(u)$ and $u \neq 0$. From (3.13), we obtain

$$
\begin{aligned}
\|u\|_{L^{2}\left(L^{1}\right)} & =\int_{\Omega_{T}} \lambda u \mathrm{~d} x \mathrm{~d} t \leq \int_{0}^{T}\|\lambda(t)\|_{L^{\infty}(\Omega)}\|u(t)\|_{L^{1}(\Omega)} \mathrm{d} t \\
& \leq\left(\int_{0}^{T}\|\lambda(t)\|_{L^{\infty}(\Omega)}^{2} \mathrm{~d} t\right)^{1 / 2}\left(\int_{0}^{T}\|u(t)\|_{L^{1}(\Omega)}^{2} \mathrm{~d} t\right)^{1 / 2} \\
& =\|\lambda\|_{L^{2}\left(L^{\infty}\right)}\|u\|_{L^{2}\left(L^{1}\right)} \leq\|u\|_{L^{2}\left(L^{1}\right)},
\end{aligned}
$$

hence

$$
\int_{0}^{T}\|\lambda(t)\|_{L^{\infty}(\Omega)}\|u(t)\|_{L^{1}(\Omega)} \mathrm{d} t=\left(\int_{0}^{T}\|\lambda(t)\|_{L^{\infty}(\Omega)}^{2} \mathrm{~d} t\right)^{1 / 2}\left(\int_{0}^{T}\|u(t)\|_{L^{1}(\Omega)}^{2} \mathrm{~d} t\right)^{1 / 2} .
$$

Therefore there exists a constant $c>0$ such that $\|\lambda(t)\|_{L^{\infty}(\Omega)}=c\|u(t)\|_{L^{1}(\Omega)}$ for almost all $t \in(0, T)$. We infer from (3.15) that $\|\lambda\|_{L^{2}\left(L^{\infty}\right)}=1$ holds, and thus we obtain $c=\|u\|_{L^{2}\left(L^{1}\right)}^{-1}$. From (3.14) we conclude $\int_{\Omega} \lambda(x, t) u(x, t) \mathrm{d} x=\|\lambda(t)\|_{L^{\infty}}\|u(t)\|_{L^{1}}$. This shows (3.10).

The characterization of $\partial j_{2}(0)$ follows directly from (3.13). Finally, (3.12) follows from (3.10).

Now, we compute the directional derivatives $j_{2}^{\prime}(u ; v)$. First, we define the auxiliary functional

$$
j_{\Omega}: L^{1}(\Omega) \rightarrow \mathbb{R}, \quad j_{\Omega}(u)=\int_{\Omega}|u(x)| \mathrm{d} x
$$

Analogously to (3.6), we obtain that the directional derivative of $j_{\Omega}$ is given by

$$
j_{\Omega}^{\prime}(u ; v)=\int_{\Omega_{u}^{+}} v(x) \mathrm{d} x-\int_{\Omega_{u}^{-}} v(x) \mathrm{d} x+\int_{\Omega_{u}^{0}}|v(x)| \mathrm{d} x .
$$

Note that

$$
j_{2}(u)=\left(\int_{0}^{T} j_{\Omega}(u(t))^{2} \mathrm{~d} t\right)^{1 / 2}
$$

Proposition 3.5. For every $u, v \in L^{2}\left(0, T ; L^{1}(\Omega)\right)$ we have either

$$
j_{2}^{\prime}(u ; v)=\frac{1}{\|u\|_{L^{2}\left(L^{1}\right)}} \int_{0}^{T} j_{\Omega}^{\prime}(u(t) ; v(t))\|u(t)\|_{L^{1}(\Omega)} \mathrm{d} t
$$

in case $u \neq 0$ or

$$
j_{2}^{\prime}(u ; v)=j_{2}(v)
$$

in case $u=0$. 
Proof. Since $j_{2}$ is positively homogeneous, (3.19) is obvious and we need to consider only the case $u \neq 0$. Let us take $0<\rho<1$, then

$$
\begin{aligned}
\frac{j_{2}(u+\rho v)-j_{2}(u)}{\rho} & =\frac{1}{\rho}\left(\|u+\rho v\|_{L^{2}\left(L^{1}\right)}-\|u\|_{L^{2}\left(L^{1}\right)}\right)=\frac{1}{\rho} \frac{\|u+\rho v\|_{L^{2}\left(L^{1}\right)}^{2}-\|u\|_{L^{2}\left(L^{1}\right)}^{2}}{\|u+\rho v\|_{L^{2}\left(L^{1}\right)}+\|u\|_{L^{2}\left(L^{1}\right)}} \\
& =\frac{\int_{0}^{T}\left[j_{\Omega}(u(t)+\rho v(t))+j_{\Omega}(u(t))\right] \frac{1}{\rho}\left[j_{\Omega}(u(t)+\rho v(t))-j_{\Omega}(u(t))\right] \mathrm{d} t}{\|u+\rho v\|_{L^{2}\left(L^{1}\right)}+\|u\|_{L^{2}\left(L^{1}\right)}} .
\end{aligned}
$$

It is enough to take the limit when $\rho \searrow 0$ to deduce (3.18).

Now, we deduce from Theorem 3.1 the following corollary in the case $j=j_{2}$.

Corollary 3.6. Let $\bar{u}_{\nu}, \bar{\varphi}_{\nu}$ and $\bar{\lambda}_{\nu}$ be as in Theorem 3.1, then the following relations hold for almost all $(x, t) \in \Omega_{T}$

$$
\begin{aligned}
& \text { if } \nu>0 \quad\left\{\begin{array}{l}
\bar{u}_{\nu}(x, t)=\operatorname{Proj}_{[\alpha, \beta]}\left(-\frac{1}{\nu}\left[\bar{\varphi}_{\nu}(x, t)+\mu \bar{\lambda}_{\nu}(x, t)\right]\right), \\
\bar{u}_{\nu}(x, t)=0 \Leftrightarrow\left|\bar{\varphi}_{\nu}(x, t)\right| \leq \mu \bar{\gamma}_{\nu}(t),
\end{array}\right. \\
& \text { if } \nu=0 \quad\left\{\begin{array}{l}
\left|\bar{\varphi}_{\nu}(x, t)\right|<\mu \bar{\gamma}_{\nu}(t) \Rightarrow \bar{u}_{\nu}(x, t)=0, \\
\bar{\varphi}_{\nu}(x, t)>+\mu \bar{\gamma}_{\nu}(t) \Rightarrow \bar{u}_{\nu}(x, t)=\alpha, \\
\bar{\varphi}_{\nu}(x, t)<-\mu \bar{\gamma}_{\nu}(t) \Rightarrow \bar{u}_{\nu}(x, t)=\beta,
\end{array}\right. \\
& \bar{\lambda}_{\nu}(x, t)=\operatorname{Proj}_{\left[-\bar{\gamma}_{\nu}(t),+\bar{\gamma}_{\nu}(t)\right]}\left(-\frac{1}{\mu} \bar{\varphi}_{\nu}(x, t)\right),
\end{aligned}
$$

where

$$
\bar{\gamma}_{\nu}(t)= \begin{cases}\frac{1}{\left\|\bar{u}_{\nu}\right\|_{L^{2}\left(L^{1}\right)}}\left\|\bar{u}_{\nu}(t)\right\|_{L^{1}(\Omega)} & \text { if } \bar{u}_{\nu} \neq 0 \\ 1 & \text { if } \bar{u}_{\nu}=0 .\end{cases}
$$

Moreover, $\bar{\lambda}_{\nu} \in L^{2}\left(0, T ; H_{0}^{1}(\Omega)\right)$ holds and it is unique. Finally, if $\nu>0$, we also have that $\bar{u}_{\nu} \in L^{2}\left(0, T ; H_{0}^{1}(\Omega)\right)$.

Proof. Let us assume that $\bar{u}_{\nu} \neq 0$ and $\nu>0$. The first identity of (3.20) is a straightforward consequence of (3.4). Let us prove the second relation of (3.20). First, we assume that $\bar{u}_{\nu}(x, t)=0$, then from the first identity we deduce that $\bar{\varphi}_{\nu}(x, t)=-\mu \bar{\lambda}_{\nu}(x, t)$. Hence, (3.10) implies that $\left|\bar{\varphi}_{\nu}(x, t)\right|=\left|\mu \bar{\lambda}_{\nu}(x, t)\right| \leq \mu \bar{\gamma}_{\nu}(t)$. To prove the converse implication we proceed as follows. Let us assume that $\bar{u}_{\nu}(x, t)>0$. Then, from the first equality of (3.20) we get that $\bar{\varphi}_{\nu}(x, t)+\mu \bar{\lambda}_{\nu}(x, t)<0$. This inequality and (3.10) leads to $\bar{\varphi}_{\nu}(x, t)<$ $-\mu \bar{\lambda}_{\nu}(x, t)=-\mu \bar{\gamma}_{\nu}(t)$. Therefore, we get $\left|\bar{\varphi}_{\nu}(x, t)\right|>\mu \bar{\gamma}_{\nu}(t)$. Analogously we prove $\left|\bar{\varphi}_{\nu}(x, t)\right|>\mu \bar{\gamma}_{\nu}(t)$ in case $\bar{u}_{\nu}(x, t)<0$. Consequently, $\left|\bar{\varphi}_{\nu}(x, t)\right| \leq \mu \bar{\gamma}_{\nu}(t)$ implies that $\bar{u}_{\nu}(x, t)=0$.

We still assume that $\bar{u}_{\nu} \neq 0$ and $\nu>0$. Let us prove (3.22). If $-\frac{1}{\mu} \bar{\varphi}_{\nu}(x, t) \in\left[-\bar{\gamma}_{\nu}(t),+\bar{\gamma}_{\nu}(t)\right]$, then the second relation of (3.20) implies that $\bar{u}_{\nu}(x, t)=0$, and from the first relation it follows that $\bar{\varphi}_{\nu}(x, t)+\mu \bar{\lambda}_{\nu}(x, t)=0$, which implies (3.22). If $-\frac{1}{\mu} \bar{\varphi}_{\nu}(x, t)>\bar{\gamma}_{\nu}(t)$, then with (3.10) we deduce that $\bar{\varphi}_{\nu}(x, t)+\mu \bar{\lambda}_{\nu}(x, t) \leq \bar{\varphi}_{\nu}(x, t)+$ $\mu \bar{\gamma}_{\nu}(t)<0$. Hence, from the first formula of $(3.20)$ we get that $\bar{u}_{\nu}(x, t)>0$. Then, (3.10) leads to $\bar{\lambda}_{\nu}(x, t)=\bar{\gamma}_{\nu}(t)$, and the projection formula $(3.22)$ holds. Similarly we proceed for the case $-\frac{1}{\mu} \bar{\varphi}_{\nu}(x, t)<-\bar{\gamma}_{\nu}(t)$.

Now, we analyze the case $\bar{u}_{\nu} \neq 0$ and $\nu=0$. First, we prove (3.21). Using once again (3.10), we have that $\left|\bar{\lambda}_{\nu}(x, t)\right| \leq \bar{\gamma}_{\nu}(t)$. Then, $\bar{\varphi}_{\nu}(x, t)>\mu \bar{\gamma}_{\nu}(t)$ implies that $\bar{\varphi}_{\nu}(x, t)>-\mu \bar{\lambda}_{\nu}(x, t)$, therefore $\bar{\varphi}_{\nu}(x, t)+\mu \bar{\lambda}_{\nu}(x, t)>0$. 
From this inequality and (3.4) we infer that $\bar{u}_{\nu}(x, t)=\alpha$. Analogously we prove that $\bar{u}_{\nu}(x, t)=\beta$ if $\bar{\varphi}_{\nu}(x, t)<$ $-\mu \bar{\gamma}_{\nu}(t)$. To prove the first inequality of (3.21), we first observe that (3.4) implies

$$
\bar{u}_{\nu}(x, t)=\left\{\begin{array}{l}
\alpha \text { if } \bar{\varphi}_{\nu}(x, t)+\mu \bar{\lambda}_{\nu}(x, t)>0, \\
\beta \text { if } \bar{\varphi}_{\nu}(x, t)+\mu \bar{\lambda}_{\nu}(x, t)<0 .
\end{array}\right.
$$

Now, if $\left|\bar{\varphi}_{\nu}(x, t)\right|<\bar{\gamma}_{\nu}(t)$ and $\bar{u}_{\nu}(x, t)>0$, we obtain from (3.10) that $\bar{\varphi}_{\nu}(x, t)+\mu \bar{\lambda}_{\nu}(x, t)>0$. Then, (3.23) leads to the contradiction that $\bar{u}_{\nu}(x, t)=\alpha<0$. Analogously we argue if $\bar{u}_{\nu}(x, t)<0$. All these arguments imply the first relation of (3.21).

Finally, we prove (3.22) under the assumption $\bar{u}_{\nu} \neq 0$ and $\nu=0$. First, let us consider the case $-\frac{1}{\mu} \bar{\varphi}_{\nu}(x, t)>$ $\bar{\gamma}_{\nu}(t)$. Then, $\bar{\varphi}_{\nu}(x, t)<-\mu \bar{\gamma}_{\nu}(t)$, hence the third relation of (3.21) implies that $\bar{u}_{\nu}(x, t)=\beta$. From (3.10) the identity $\bar{\lambda}_{\nu}(x, t)=\bar{\gamma}_{\nu}(t)$ follows. Therefore, (3.22) holds in this case. Analogously we proceed for the case $-\frac{1}{\mu} \bar{\varphi}_{\nu}(x, t)<-\bar{\gamma}_{\nu}(t)$. Now, we consider the last case $-\frac{1}{\mu} \bar{\varphi}_{\nu}(x, t) \in\left[-\bar{\gamma}_{\nu}(t),+\bar{\gamma}_{\nu}(t)\right]$. Under this assumption, if in addition $\bar{u}_{\nu}(x, t)=\alpha$, then (3.10) and (3.23) imply that $\bar{\lambda}_{\nu}(x, t)=-\bar{\gamma}_{\nu}(t)$ and $\bar{\varphi}_{\nu}(x, t)+\mu \bar{\lambda}_{\nu}(x, t) \geq 0$. Thus, we obtain

$$
-\bar{\gamma}_{\nu}(t) \leq-\frac{1}{\mu} \bar{\varphi}_{\nu}(x, t) \leq \bar{\lambda}_{\nu}(x, t)=-\bar{\gamma}_{\nu}(t) .
$$

Hence, $\bar{\lambda}_{\nu}(x, t)=-\frac{1}{\mu} \bar{\varphi}_{\nu}(x, t)$ and (3.22) holds. In the same way we prove this equality if $\bar{u}_{\nu}(x, t)=\beta$. Finally, according to (3.23), if $\alpha<\bar{u}_{\nu}(x, t)<\beta$, then $\bar{\lambda}_{\nu}(x, t)=-\frac{1}{\mu} \bar{\varphi}_{\nu}(x, t)$ and (3.22) holds as well.

In the case $\bar{u}_{\nu}=0$ we can follow the same steps as above and replace the invocation of (3.10) by $\|\bar{\lambda}\|_{L^{2}\left(L^{\infty}\right)} \leq 1$. Note that some of the implications in (3.20) and (3.21) become trivial in this case.

The regularity properties of $\bar{\lambda}_{\nu}$ and $\bar{u}_{\nu}$ are immediate consequences of (3.22) and (3.20), respectively.

Remark 3.7. Let us observe that $\bar{u}_{\nu}=0$ if $\mu$ is bigger than a certain value $\mu_{0}$. Indeed, as pointed out in Remark 3.3, there exists a constant $M^{\prime}>0$ such that $\left\|\bar{\varphi}_{\nu}\right\|_{L^{\infty}\left(\Omega_{T}\right)} \leq M^{\prime}$ with $M^{\prime}$ depending on $\alpha$ and $\beta$, but independent of $\mu$. If we take $\mu_{0}=M^{\prime} \sqrt{T}$, then $\bar{u}_{\nu}=0$ for every $\mu>\mu_{0}$. Let us prove it by contradiction. If $\bar{u}_{\nu} \neq 0$, then the identity (3.22) holds, and consequently

$$
\left\|\bar{\lambda}_{\nu}\right\|_{L^{2}\left(L^{\infty}\right)} \leq \frac{1}{\mu}\left\|\bar{\varphi}_{\nu}\right\|_{L^{2}\left(L^{\infty}\right)} \leq \frac{M^{\prime} \sqrt{T}}{\mu}=\frac{\mu_{0}}{\mu}<1 .
$$

But, (3.10) and the above inequality imply that $\bar{u}_{\nu}=0$. Therefore, we may influence the size of an optimal control's support by adjusting $\mu$ in the interval $\left[0, M^{\prime} \sqrt{T}\right]$.

\subsection{Problem $\left(\mathbf{P}_{\nu}^{3}\right)$}

Now, we consider the functional $j_{3}: L^{1}\left(\Omega ; L^{2}(0, T)\right) \rightarrow \mathbb{R}$ given by

$$
j_{3}(u)=\int_{\Omega}\|u(x)\|_{L^{2}(0, T)} \mathrm{d} x=\int_{\Omega}\left(\int_{0}^{T} u(x, t)^{2} \mathrm{~d} t\right)^{1 / 2} \mathrm{~d} x .
$$

Let us study the properties of this functional. To this end, we introduce a new functional that will be used later in this paper. Let $\Psi: L^{2}(0, T) \rightarrow \mathbb{R}$ be defined by $\Psi(f)=\|f\|_{L^{2}(0, T)}$. This functional is of class $C^{\infty}$ in $L^{2}(0, T) \backslash\{0\}$ and we have for every $f, g \in L^{2}(0, T)$, with $f \neq 0$,

$$
\begin{aligned}
\Psi^{\prime}(f) g & =\frac{1}{\|f\|_{L^{2}(0, T)}} \int_{0}^{T} f g \mathrm{~d} t, \\
\Psi^{\prime \prime}(f) g^{2} & =\frac{1}{\|f\|_{L^{2}(0, T)}}\left\{\int_{0}^{T} g^{2} \mathrm{~d} t-\frac{1}{\|f\|_{L^{2}(0, T)}^{2}}\left(\int_{0}^{T} f g \mathrm{~d} t\right)^{2}\right\}, \\
\Psi^{\prime \prime \prime}(f) g^{3} & =\frac{3}{\|f\|_{L^{2}(0, T)}^{3}}\left\{\frac{1}{\|f\|_{L^{2}(0, T)}^{2}}\left(\int_{0}^{T} f g \mathrm{~d} t\right)^{3}-\left(\int_{0}^{T} g^{2} \mathrm{~d} t\right)\left(\int_{0}^{T} f g \mathrm{~d} t\right)\right\} .
\end{aligned}
$$


Given an element $u \in L^{1}\left(\Omega ; L^{2}(0, T)\right)$, we denote

$$
\Omega_{u}=\left\{x \in \Omega:\|u(x)\|_{L^{2}(0, T)} \neq 0\right\} \text { and } \Omega_{u}^{0}=\Omega \backslash \Omega_{u} .
$$

Now we characterize $\partial j_{3}(u)$ and compute $j_{3}^{\prime}(u ; v)$.

Proposition 3.8. The following statements hold.

(1) $\lambda \in \partial j_{3}(u)$ is equivalent to $\lambda \in L^{\infty}\left(\Omega ; L^{2}(0, T)\right)$ and

$$
\left\{\begin{array}{l}
\|\lambda(x)\|_{L^{2}(0, T)} \leq 1 \quad \text { for a.a. } x \in \Omega_{u}^{0}, \\
\lambda(x, t)=\frac{u(x, t)}{\|u(x)\|_{L^{2}(0, T)}} \text { for a.a. } x \in \Omega_{u} \text { and } t \in(0, T) .
\end{array}\right.
$$

(2) For every $u, v \in L^{1}\left(\Omega ; L^{2}(0, T)\right)$

$$
j_{3}^{\prime}(u ; v)=\int_{\Omega_{u}^{0}}\|v(x)\|_{L^{2}(0, T)} \mathrm{d} x+\int_{\Omega_{u}} \frac{1}{\|u(x)\|_{L^{2}(0, T)}} \int_{0}^{T} u v \mathrm{~d} t \mathrm{~d} x .
$$

Proof. Since $L^{\infty}\left(\Omega ; L^{2}(0, T)\right)$ is the dual space of $L^{1}\left(\Omega ; L^{2}(0, T)\right)$, we have, similarly to (3.13), the characterization

$$
\partial j_{3}(u)=\left\{\lambda \in L^{\infty}\left(\Omega ; L^{2}(0, T)\right):\|\lambda\|_{L^{\infty}\left(L^{2}\right)} \leq 1 \text { and } \int_{\Omega_{T}} \lambda u \mathrm{~d} x \mathrm{~d} t=\|u\|_{L^{1}\left(L^{2}\right)}\right\} .
$$

If $\lambda \in \partial j_{3}(u)$, the first relation of (3.27) is an obvious consequence of (3.29). Let us prove the identity of (3.27). Since

$$
\|u\|_{L^{1}\left(L^{2}\right)}=\int_{\Omega_{T}} \lambda u \mathrm{~d} x \mathrm{~d} t \leq \int_{\Omega}\|\lambda(x)\|_{L^{2}(0, T)}\|u(x)\|_{L^{2}(0, T)} \mathrm{d} x \leq\|u\|_{L^{1}\left(L^{2}\right)},
$$

we find $\|\lambda(x)\|_{L^{2}(0, T)}=1$ and $\lambda(x, t)=c(x) u(x, t)$ with $c(x)>0$ for a.a. $x \in \Omega_{u}$ and $t \in(0, T)$. Now, (3.27) follows.

Conversely, suppose $\lambda$ satisfies (3.27). Using (3.29), it is easy to check $\lambda \in \partial j_{3}(u)$.

Finally (3.28) is obtained with (3.24) as follows

$$
\begin{gathered}
\lim _{\rho \searrow 0} \frac{j_{3}(u+\rho v)-j_{3}(u)}{\rho}=\int_{\Omega_{u}^{0}}\|v(x)\|_{L^{2}(0, T)} \mathrm{d} x+\lim _{\rho \searrow 0} \int_{\Omega_{u}} \frac{\Psi(u+\rho v)-\Psi(u)}{\rho} \mathrm{d} x \\
=\int_{\Omega_{u}^{0}}\|v(x)\|_{L^{2}(0, T)} \mathrm{d} x+\int_{\Omega_{u}} \frac{1}{\|u(x)\|_{L^{2}(0, T)}} \int_{0}^{T} u v \mathrm{~d} t \mathrm{~d} x .
\end{gathered}
$$

Corollary 3.9. Let $\bar{u}_{\nu}, \bar{\varphi}_{\nu}$ and $\bar{\lambda}_{\nu}$ be as in Theorem 3.1, then the following relations hold for almost all $(x, t) \in \Omega_{T}$

$$
\begin{gathered}
\text { if } \nu>0 \quad\left\{\begin{array}{l}
\bar{u}_{\nu}(x, t)=\operatorname{Proj}_{[\alpha, \beta]}\left(-\frac{1}{\nu}\left[\bar{\varphi}_{\nu}(x, t)+\mu \bar{\lambda}_{\nu}(x, t)\right]\right), \\
\left\|\bar{u}_{\nu}(x)\right\|_{L^{2}(0, T)}=0 \Leftrightarrow\left\|\bar{\varphi}_{\nu}(x)\right\|_{L^{2}(0, T)} \leq \mu,
\end{array}\right. \\
\text { if } \nu=0 \quad\left\{\begin{array}{l}
\left\|\bar{\varphi}_{\nu}(x)\right\|_{L^{2}(0, T)}<\mu \Rightarrow\left\|\bar{u}_{\nu}(x)\right\|_{L^{2}(0, T)}=0 \\
\left\|\bar{u}_{\nu}(x)\right\|_{L^{2}(0, T)}=0 \Rightarrow\left\|\bar{\varphi}_{\nu}(x)\right\|_{L^{2}(0, T)} \leq \mu,
\end{array}\right. \\
\bar{\lambda}_{\nu}(x, t)= \begin{cases}-\frac{1}{\mu} \bar{\varphi}_{\nu}(x, t) & \text { if } x \in \Omega_{\bar{u}_{\nu}}^{0}, \\
\frac{\bar{u}_{\nu}(x, t)}{\left\|\bar{u}_{\nu}(x)\right\|_{L^{2}(0, T)}} & \text { if } x \in \Omega_{\bar{u}_{\nu}} .\end{cases}
\end{gathered}
$$

Moreover, $\bar{\lambda}_{\nu}$ is unique. 
Proof. The first identity of (3.30) is standard. Let us prove the second relation. First we assume that $\left\|\bar{u}_{\nu}(x)\right\|_{L^{2}(0, T)}=0$, then (3.30) implies that $\bar{\varphi}_{\nu}(x, t)+\mu \bar{\lambda}_{\nu}(x, t)=0$ for almost all $t \in(0, T)$. Hence, using (3.27) we get $\left\|\bar{\varphi}_{\nu}(x)\right\|_{L^{2}(0, T)} \leq \mu\left\|\bar{\lambda}_{\nu}(x)\right\|_{L^{2}(0, T)} \leq \mu$. To prove the converse implication we introduce the set

$$
E=\left\{x \in \Omega:\left\|\bar{\varphi}_{\nu}(x)\right\|_{L^{2}(0, T)} \leq \mu \text { and }\left\|\bar{u}_{\nu}(x)\right\|_{L^{2}(0, T)} \neq 0\right\} .
$$

We have to prove that $|E|=0$. Let us take in (3.4)

$$
v(x, t)=\left\{\begin{array}{cl}
-\bar{u}_{\nu}(x, t) & \text { if } x \in E \\
0 & \text { otherwise. }
\end{array}\right.
$$

Note that $v \in \mathcal{T}_{\mathbb{K}}\left(\bar{u}_{\nu}\right)$ holds in view of $\alpha<0$ and $\beta>0$. Then we have

$$
\int_{E} \int_{0}^{T}\left(\bar{\varphi}_{\nu}+\mu \bar{\lambda}_{\nu}+\nu \bar{u}_{\nu}\right) \bar{u}_{\nu} \mathrm{d} t \mathrm{~d} x \leq 0 .
$$

Using the second relation of (3.27), we deduce from the above inequality

$$
\begin{aligned}
& \mu \int_{E}\left\|\bar{u}_{\nu}(x)\right\|_{L^{2}(0, T)} \mathrm{d} x+\nu \int_{E}\left\|\bar{u}_{\nu}(x)\right\|_{L^{2}(0, T)}^{2} \mathrm{~d} x \leq-\int_{E} \int_{0}^{T} \bar{\varphi}_{\nu} \bar{u}_{\nu} \mathrm{d} t \mathrm{~d} x \\
& \quad \leq \int_{E}\left\|\bar{\varphi}_{\nu}(x)\right\|_{L^{2}(0, T)}\left\|\bar{u}_{\nu}(x)\right\|_{L^{2}(0, T)} \mathrm{d} x \leq \mu \int_{E}\left\|\bar{u}_{\nu}(x)\right\|_{L^{2}(0, T)} \mathrm{d} x .
\end{aligned}
$$

Since $\nu>0$, the above inequality is possible only if $|E|=0$.

Let us consider the case $\nu=0$. The second implication of (3.31) is proved as the corresponding implication to the case $\nu>0$. The first implication is also proved arguing as above, the only difference is that the identity $|E|=0$ does not follow from $\nu>0$, but from the strict inequality $\left\|\bar{\varphi}_{\nu}(x)\right\|_{L^{2}(0, T)}<\mu$.

Finally, (3.32) is an immediate consequence of (3.4) and (3.27). The uniqueness of $\bar{\lambda}_{\nu}$ follows from the representation (3.32).

Remark 3.10. As in Remarks 3.3 and 3.7, we can obtain the existence of a constant $M>0$ independent of $\mu$ such that $\left\|\bar{\varphi}_{\nu}\right\|_{L^{\infty}\left(L^{2}\right)} \leq M$. Therefore, (3.30) and (3.31) imply that $\bar{u}_{\nu}=0$ if $\mu>M$. Hence, we can influence the size of of an optimal control's support by adjusting the parameter $\mu \in[0, M]$.

Remark 3.11. It is interesting to compare the sparsity properties of the local solutions $\bar{u}_{\nu}$ corresponding to the studied problems. From (3.7) and (3.8) we obtain that the local solutions $\bar{u}_{\nu}$ of $\left(\mathrm{P}_{\nu}^{1}\right)$ are sparse in space and time. However, the solutions of $\left(\mathrm{P}_{\nu}^{3}\right)$ are only sparse in space as proved by (3.30) and (3.31), the sparsity region remaining constant throughout time. When we look at (3.20) and (3.21), we observe that the sparsity region of the solutions of $\left(\mathrm{P}_{\nu}^{2}\right)$ can change with the time. Thus we confirm the sparsity patterns as anticipated in the introduction. Any of the three formulations can be interesting with different possible applications.

In Figure 1, we show the optimal controls in the linear case using $T=1, \Omega=(0,1)$ and the parameters

$$
\begin{array}{llrl}
\alpha & =-10, & \beta & =20, \\
\nu & =1 e-4, & \mu & =4 e-3 .
\end{array}
$$

The objective is $F_{\nu}(u)=\frac{1}{2}\left\|y_{u}-y_{d}\right\|_{L^{2}\left(\Omega_{T}\right)}^{2}+\frac{\nu}{2}\|u\|_{L^{2}\left(\Omega_{T}\right)}^{2}$, where the desired state is given by

$$
y_{d}(x, t)=\exp \left(-20\left[(x-0.2)^{2}+(t-0.2)^{2}\right]\right)+\exp \left(-20\left[(x-0.7)^{2}+(t-0.9)^{2}\right]\right) .
$$

The state equation is the one-dimensional linear parabolic equation

$$
\partial_{t} y_{u}-\Delta y_{u}=u \quad \text { in } \Omega_{T}
$$

with homogeneous initial and Dirichlet boundary conditions. 

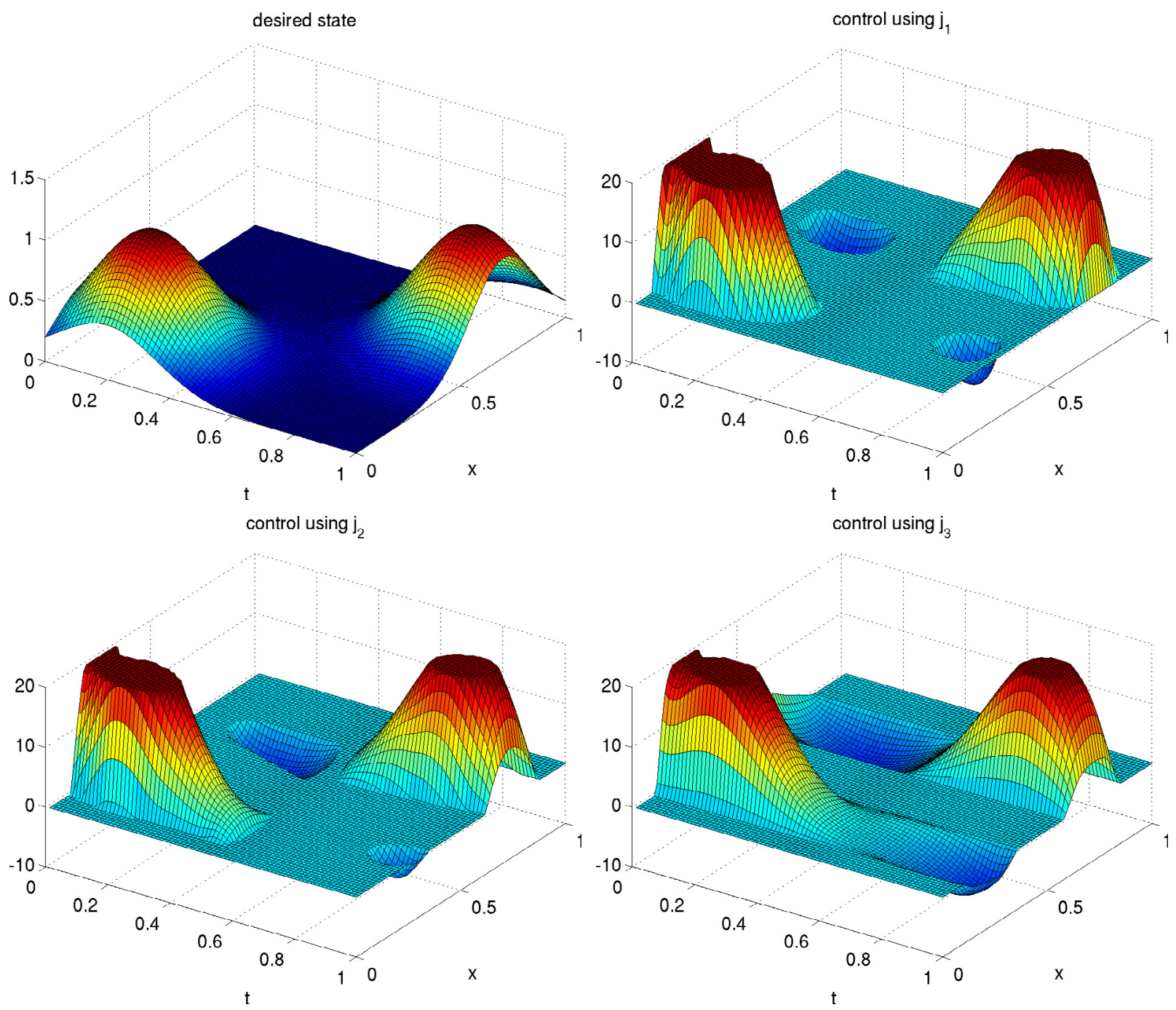

FiguRE 1. Different sparsity structures of optimal controls using $j_{1}$ (top right), $j_{2}$ (bottom left) and $j_{3}$ (bottom right). The desired state is shown top left. The problem parameters are given in Remark 3.11.

\section{SECOND-ORDER NECESSARY OPTIMALITY CONDITIONS}

In this section, $\bar{u}_{\nu}$ denotes an element of $\mathbb{K}$, with associated elements $\left(\bar{y}_{\nu}, \bar{\varphi}_{\nu}, \bar{\lambda}_{\nu}\right) \in Y \times \Phi \times \partial j\left(\bar{u}_{\nu}\right)$, such that the optimality system (3.2)-(3.4) holds. In order to address the necessary second-order optimality conditions, we introduce the cone of critical directions as follows.

$$
C_{\bar{u}_{\nu}}=\left\{v \in \mathcal{T}_{\mathbb{K}}\left(\bar{u}_{\nu}\right): F_{\nu}^{\prime}\left(\bar{u}_{\nu}\right) v+\mu j^{\prime}\left(\bar{u}_{\nu} ; v\right)=0\right\} .
$$

Proposition 4.1. The set $C_{\bar{u}_{\nu}}$ is a closed, convex cone in $L^{2}\left(\Omega_{T}\right)$.

Before proving this proposition we have to establish the following lemma. 
Lemma 4.2. Let $v \in \mathcal{T}_{\mathbb{K}}\left(\bar{u}_{\nu}\right)$ be given. Then,

$$
F_{\nu}^{\prime}\left(\bar{u}_{\nu}\right) v+\mu j^{\prime}\left(\bar{u}_{\nu} ; v\right) \geq F_{\nu}^{\prime}\left(\bar{u}_{\nu}\right) v+\mu \int_{\Omega_{T}} \bar{\lambda}_{\nu}(x, t) v(x, t) \mathrm{d} x \mathrm{~d} t \geq 0 .
$$

Moreover, if $v \in C_{\bar{u}_{\nu}}$, then

$$
F_{\nu}^{\prime}\left(\bar{u}_{\nu}\right) v+\mu \int_{\Omega_{T}} \bar{\lambda}_{\nu}(x, t) v(x, t) \mathrm{d} x \mathrm{~d} t=0 \quad \text { and } \quad j^{\prime}\left(\bar{u}_{\nu} ; v\right)=\int_{\Omega_{T}} \bar{\lambda}_{\nu}(x, t) v(x, t) \mathrm{d} x \mathrm{~d} t .
$$

Proof. It is well-known (see [1], Prop. 2.126(v)), that for any convex and Lipschitz function the following inequality holds

$$
\max _{\lambda \in \partial j(u)} \int_{\Omega_{T}} \lambda v \mathrm{~d} x \mathrm{~d} t=j^{\prime}(u ; v) \leq \frac{j(u+\rho v)-j(u)}{\rho} \quad \forall 0<\rho \leq 1 .
$$

Hence, the first inequality of (4.2) is an immediate consequence of (4.4). The second inequality follows from (3.4) and (2.9). Identities (4.3) are an obvious consequence of (4.2) and the definition (4.1) of $C_{\bar{u}_{\nu}}$.

Proof of Proposition 4.1. It is obvious that $C_{\bar{u}_{\nu}}$ is a closed cone of $L^{2}\left(\Omega_{T}\right)$, thanks to the continuity of $v \mapsto$ $j^{\prime}(u ; v)$. Let us prove that it is convex. Given $v_{1}, v_{2} \in C_{\bar{u}_{\nu}}$ and $0<t<1$, it is clear that $v=t v_{1}+(1-t) v_{2} \in$ $\mathcal{T}_{\mathbb{K}}\left(\bar{u}_{\nu}\right)$. Moreover, using the convexity of $v \rightarrow j^{\prime}\left(\bar{u}_{\nu} ; v\right)$ we get

$$
F_{\nu}^{\prime}\left(\bar{u}_{\nu}\right) v+\mu j\left(\bar{u}_{\nu} ; v\right) \leq t\left[F_{\nu}^{\prime}\left(\bar{u}_{\nu}\right) v_{1}+\mu j^{\prime}\left(\bar{u}_{\nu} ; v_{1}\right)\right]+(1-t)\left[F_{\nu}^{\prime}\left(\bar{u}_{\nu}\right) v_{2}+\mu j^{\prime}\left(\bar{u}_{\nu} ; v_{2}\right)\right]=0 .
$$

The contrary inequality is a consequence of Lemma 4.2 , hence $v \in C_{\bar{u}_{\nu}}$.

Now, we are going to define replacements for the second directional derivatives of the functional $j$, denoted by $j^{\prime \prime}$, which are obtained by formal calculations. Note that the symbol $j^{\prime \prime}$ does not mean that the respective terms are second-order directional derivatives. Indeed, those derivatives do not exist for all directions $v$. Given $u, v \in L^{2}\left(\Omega_{T}\right)$, we set $J_{\nu}^{\prime \prime}\left(u ; v^{2}\right)=F_{\nu}^{\prime \prime}(u) v^{2}+\mu j^{\prime \prime}\left(u ; v^{2}\right)$, with $F_{\nu}^{\prime \prime}(u)$ defined by $(2.10)$ and $j^{\prime \prime}\left(u ; v^{2}\right)$ is defined for the three different functionals under investigation

$$
\begin{aligned}
& j_{1}^{\prime \prime}\left(u ; v^{2}\right)=0 \\
& j_{2}^{\prime \prime}\left(u ; v^{2}\right)= \begin{cases}\frac{1}{\|u\|_{L^{2}\left(L^{1}\right)}}\left(\int_{0}^{T} j_{\Omega}^{\prime}(u(t) ; v(t))^{2} \mathrm{~d} t-j_{2}^{\prime}(u ; v)^{2}\right) & \text { if } u \neq 0 \\
0 & \text { if } u=0\end{cases} \\
& j_{3}^{\prime \prime}\left(u ; v^{2}\right)= \begin{cases}\int_{\Omega_{u}} \frac{1}{\|u(x)\|_{L^{2}(0, T)}}\left[\int_{0}^{T} v^{2}(x, t) \mathrm{d} t-\left(\int_{0}^{T} \frac{u(x, t) v(x, t)}{\|u(x)\|_{L^{2}(0, T)}} \mathrm{d} t\right)^{2}\right] \mathrm{d} x & \text { if } u \neq 0 \\
0 & \text { if } u=0 .\end{cases}
\end{aligned}
$$

In (4.6) and (4.7), $j_{\Omega}^{\prime}$ is given by (3.17) and $\Omega_{u}$ is defined in Section 3.3.

With this notation, we have the following second-order necessary optimality conditions valid for the three functionals $j_{i}$.

Theorem 4.3. Let $\nu \geq 0$ and $\bar{u}_{\nu}$ be a local minimum of $\left(\mathrm{P}_{\nu}\right)$. Then $J_{\nu}^{\prime \prime}\left(\bar{u}_{\nu} ; v^{2}\right) \geq 0$ for every $v \in C_{\bar{u}_{\nu}}$.

The rest of the section will be devoted to the proof of this theorem. We distinguish three cases. 
Proof.

Case I: $j=j_{1}$. The proof for this case is exactly as the one of (Thm. 3.7 of [5]), with obvious modifications.

Case II: $j=j_{2}$. First we assume that $\bar{u}_{\nu} \neq 0$. Given $v \in C_{\bar{u}_{\nu}}$ we define for every $k \in \mathbb{N}$

$$
v_{k}(x, t)=\left\{\begin{array}{l}
0 \text { if } \bar{u}_{\nu}(x, t) \in\left(\alpha, \alpha+\frac{1}{k}\right) \cup\left(-\frac{1}{k}, 0\right) \cup\left(0,+\frac{1}{k}\right) \cup\left(\beta-\frac{1}{k}, \beta\right), \\
\operatorname{Proj}_{[-k,+k]}(v(x, t)) \text { otherwise, }
\end{array}\right.
$$

and $\rho_{k}=1 / k^{2}$. Then, we have that $\bar{u}_{\nu}+\rho v_{k} \in \mathbb{K}$ for every $0<\rho<\rho_{k}$ and $v_{k} \rightarrow v$ in $L^{2}\left(\Omega_{T}\right)$. Moreover, from (3.17) we easily get that

$$
\frac{j_{\Omega}\left(\bar{u}_{\nu}(t)+\rho v_{k}(t)\right)-j_{\Omega}\left(\bar{u}_{\nu}(t)\right)}{\rho}=j_{\Omega}^{\prime}\left(\bar{u}_{\nu}(t) ; v_{k}(t)\right) \text { for a.a. } t \in(0, T) \text { and } \forall \rho \in\left(0, \rho_{k}\right) .
$$

Equivalently we can write

$$
j_{\Omega}\left(\bar{u}_{\nu}(t)+\rho v_{k}(t)\right)=j_{\Omega}\left(\bar{u}_{\nu}(t)\right)+\rho j_{\Omega}^{\prime}\left(\bar{u}_{\nu}(t) ; v_{k}(t)\right) \text { a.e. in }(0, T) \forall \rho \in\left(0, \rho_{k}\right) .
$$

Let us prove that $v_{k} \in C_{\bar{u}_{\nu}}$. It is obvious that $v_{k} \in \mathcal{T}_{\mathbb{K}}\left(\bar{u}_{\nu}\right)$. Since $v \in C_{\bar{u}_{\nu}}$, from (3.10), (3.17), (3.18) and (4.3) we infer that (recall $\bar{u}_{\nu} \neq 0$ is assumed)

$$
\bar{\lambda}_{\nu}(x, t) v(x, t)=\left\{\begin{array}{ccc}
+\frac{\left\|\bar{u}_{\nu}(t)\right\|_{L^{1}(\Omega)}}{\left\|\bar{u}_{\nu}\right\|_{L^{2}\left(L^{1}\right)}} v(x, t) & \text { if } & \bar{u}_{\nu}(x, t)>0 \\
-\frac{\left\|\bar{u}_{\nu}(t)\right\|_{L^{1}(\Omega)}}{\left\|\bar{u}_{\nu}\right\|_{L^{2}\left(L^{1}\right)}} v(x, t) & \text { if } & \bar{u}_{\nu}(x, t)<0 \\
\frac{\left\|\bar{u}_{\nu}(t)\right\|_{L^{1}(\Omega)}}{\left\|\bar{u}_{\nu}\right\|_{L^{2}\left(L^{1}\right)}}|v(x, t)| & \text { if } & \bar{u}_{\nu}(x, t)=0 .
\end{array}\right.
$$

Taking into account the definition of $v_{k}$, we also have the same identities for $v_{k}$ replacing $v$, which implies

$$
\int_{\Omega_{T}} \bar{\lambda}_{\nu}(x, t) v_{k}(x, t) \mathrm{d} x \mathrm{~d} t=j_{2}^{\prime}\left(\bar{u}_{\nu} ; v_{k}\right) .
$$

Observe that (3.4) implies that

$$
\left(\bar{\varphi}_{\nu}+\nu \bar{u}_{\nu}+\mu \bar{\lambda}_{\nu}\right)(x, t)\left\{\begin{array}{c}
\geq 0 \text { if } \bar{u}_{\nu}(x, t)=\alpha \\
\leq 0 \text { if } \bar{u}_{\nu}(x, t)=\beta \\
0 \text { if } \alpha<\bar{u}(x, t)<\beta .
\end{array}\right.
$$

Therefore, (4.3) and (4.9) yield $\left(\bar{\varphi}_{\nu}+\nu \bar{u}_{\nu}+\mu \bar{\lambda}_{\nu}\right) v=0$. Hence, we also have that $\left(\bar{\varphi}_{\nu}+\nu \bar{u}_{\nu}+\mu \bar{\lambda}_{\nu}\right) v_{k}=0$. All together implies that

$$
F_{\nu}^{\prime}\left(\bar{u}_{\nu}\right) v_{k}+\mu j_{2}^{\prime}\left(\bar{u}_{\nu} ; v_{k}\right)=\int_{\Omega_{T}}\left(\bar{\varphi}_{\nu}+\nu \bar{u}_{\nu}+\mu \bar{\lambda}_{\nu}\right) v_{k} \mathrm{~d} x \mathrm{~d} t=0 .
$$

Thus, we have that $v_{k} \in C_{\bar{u}_{\nu}}$. Now, using (3.24) and (3.25), with $f(t)=\left\|\bar{u}_{\nu}(t)\right\|_{L^{1}(\Omega)} \not \equiv 0$ and $g(t)=$ $j_{\Omega}\left(\bar{u}_{\nu}(t) ; v_{k}(t)\right)$, and taking into account (4.8), (3.18) and (4.6), we obtain

$$
\begin{aligned}
j_{2}\left(\bar{u}+\rho v_{k}\right) & =\Psi(f+\rho g)=\Psi(f)+\rho \Psi^{\prime}(f) g+\frac{\rho^{2}}{2} \Psi^{\prime \prime}(f+\theta \rho g) g^{2} \\
& =j_{2}\left(\bar{u}_{\nu}\right)+\rho j_{2}^{\prime}\left(\bar{u}_{\nu}, v_{k}\right)+\frac{\rho^{2}}{2} j_{2}^{\prime \prime}\left(\bar{u}_{\nu}+\theta \rho v_{k} ; v_{k}^{2}\right) .
\end{aligned}
$$


Here $\theta \in(0,1)$ depends on $k$. Using this identity and making a Taylor expansion of $F_{\nu}\left(\bar{u}_{\nu}+\rho v_{k}\right)$ we deduce for all $\rho>0$ small enough with (4.10)

$$
\begin{aligned}
0 & \leq J_{\nu}\left(\bar{u}_{\nu}+\rho v_{k}\right)-J_{\nu}\left(\bar{u}_{\nu}\right) \\
& =\rho\left\{F_{\nu}^{\prime}\left(\bar{u}_{\nu}\right) v_{k}+\mu j_{2}^{\prime}\left(\bar{u}_{\nu} ; v_{k}\right)\right\}+\frac{\rho^{2}}{2} J_{\nu}^{\prime \prime}\left(\bar{u}_{\nu}+\theta \rho v_{k} ; v_{k}^{2}\right)=\frac{\rho^{2}}{2} J_{\nu}^{\prime \prime}\left(\bar{u}_{\nu}+\theta \rho v_{k} ; v_{k}^{2}\right) .
\end{aligned}
$$

Thus, we have $J_{\nu}^{\prime \prime}\left(\bar{u}_{\nu}+\theta \rho v_{k} ; v_{k}^{2}\right) \geq 0$ for every $\rho$ small enough and all $k \in \mathbb{N}$. Taking the limit first when $\rho \rightarrow 0$ and later when $k \rightarrow \infty$, we conclude that $J_{\nu}^{\prime \prime}\left(\bar{u}_{\nu} ; v^{2}\right) \geq 0$ holds by continuity of $v \mapsto F_{\nu}^{\prime \prime}\left(\bar{u}_{\nu}\right) v^{2}$ and $v \mapsto j_{2}^{\prime \prime}\left(\bar{u}_{\nu} ; v^{2}\right)$ w.r.t. $L^{2}\left(\Omega_{T}\right)$.

Now, we consider the case $\bar{u}_{\nu}=0$. Given $v \in C_{\bar{u}_{\nu}}$ we define $v_{k}=\operatorname{Proj}_{[-k,+k]}(v) \in C_{\bar{u}_{\nu}}$ and we take $0<\rho<\min \{|\alpha|, \beta\} / k$. Then, it is easy to check that $\bar{u}_{\nu}+\rho v_{k}=\rho v_{k} \in \mathbb{K}$, and for $\rho$ small enough we have

$$
\begin{gathered}
0 \leq J_{\nu}\left(\bar{u}_{\nu}+\rho v_{k}\right)-J_{\nu}\left(\bar{u}_{\nu}\right)=F_{\nu}\left(\bar{u}_{\nu}+\rho v_{k}\right)-F_{\nu}\left(\bar{u}_{\nu}\right)+\rho j_{2}\left(v_{k}\right) \\
=\rho\left\{F_{\nu}^{\prime}\left(\bar{u}_{\nu}\right) v_{k}+j_{2}^{\prime}\left(\bar{u}_{\nu} ; v_{k}\right)\right\}+\frac{\rho^{2}}{2} F_{\nu}^{\prime \prime}\left(\bar{u}_{\nu}+\theta \rho v_{k}\right) v_{k}^{2}=F_{\nu}^{\prime \prime}\left(\bar{u}_{\nu}+\theta \rho v_{k}\right) v_{k}^{2},
\end{gathered}
$$

for some $\theta \in(0,1)$ (depending on $k$ and $\rho$ ). Hence $F_{\nu}^{\prime \prime}\left(\bar{u}_{\nu}+\theta \rho v_{k}\right) v_{k}^{2} \geq 0$. Taking the limit as $\rho \rightarrow 0$, we get $F_{\nu}^{\prime \prime}\left(\bar{u}_{\nu}\right) v_{k}^{2} \geq 0$. Finally, letting $k \rightarrow \infty$ we conclude that $J_{\nu}^{\prime \prime}\left(\bar{u}_{\nu}\right) v^{2}=F_{\nu}^{\prime \prime}\left(\bar{u}_{\nu}\right) v^{2} \geq 0$.

Case III: $j=j_{3}$. We elaborate on the proof for the case $\bar{u}_{\nu} \not \equiv 0$, the case $\bar{u}_{\nu} \equiv 0$ can be handled analogously to $j_{2}$. First, we observe that

$$
\left(\int_{0}^{T} \frac{u(x, t) v(x, t)}{\|u(x)\|_{L^{2}(0, T)}} \mathrm{d} t\right)^{2} \leq \int_{0}^{T} v^{2}(x, t) \mathrm{d} t \quad \text { for } x \in \Omega_{\bar{u}_{\nu}} .
$$

Hence, the integrand in (4.7) is nonnegative. Then, $0 \leq j_{3}^{\prime \prime}\left(\bar{u}_{\nu} ; v^{2}\right)$ is well defined for every $v \in L^{2}\left(\Omega_{T}\right)$, but it could be $+\infty$ for some directions $v$. Let us take an element $v \in C_{\bar{u}_{\nu}}$ such that

$$
\int_{\Omega_{\bar{u}_{\nu}}} \frac{\|v(x)\|_{L^{2}(0, T)}^{2}}{\left\|\bar{u}_{\nu}(x)\right\|_{L^{2}(0, T)}} \mathrm{d} x<+\infty .
$$

We will get rid of this assumption later. As in Case II, we have that $\left(\bar{\varphi}_{\nu}+\nu \bar{u}_{\nu}+\mu \bar{\lambda}_{\nu}\right) v=0$ in $\Omega_{T}$. Moreover, we have that (3.28) and (3.32) imply

$$
\begin{aligned}
0= & F_{\nu}^{\prime}\left(\bar{u}_{\nu}\right) v+\mu j_{3}^{\prime}\left(\bar{u}_{\nu} ; v\right)=\int_{\Omega_{\bar{u}_{\nu}}^{0}} \int_{0}^{T} \bar{\varphi}_{\nu} v \mathrm{~d} t \mathrm{~d} x+\mu \int_{\Omega_{\bar{u}_{\nu}}^{0}}\|v(x)\|_{L^{2}(0, T)} \mathrm{d} x \\
& +\int_{\Omega_{\bar{u}_{\nu}}} \int_{0}^{T}\left(\bar{\varphi}_{\nu}+\nu \bar{u}_{\nu}\right) v \mathrm{~d} t \mathrm{~d} x+\mu \int_{\Omega_{\bar{u}_{\nu}}} \int_{0}^{T} \frac{\bar{u}_{\nu}}{\left\|\bar{u}_{\nu}\right\|_{L^{2}(0, T)}} v \mathrm{~d} t \mathrm{~d} x \\
= & -\mu \int_{\Omega_{\bar{u}_{\nu}}^{0}} \int_{0}^{T} \bar{\lambda}_{\nu} v \mathrm{~d} t \mathrm{~d} x+\mu \int_{\Omega_{\bar{u}_{\nu}}^{0}}\|v(x)\|_{L^{2}(0, T)} \mathrm{d} x+\int_{\Omega_{\bar{u}_{\nu}}} \int_{0}^{T}\left(\bar{\varphi}_{\nu}+\nu \bar{u}_{\nu}+\mu \bar{\lambda}_{\nu}\right) v \mathrm{~d} t \mathrm{~d} x \\
= & -\mu \int_{\Omega_{\bar{u}_{\nu}}^{0}} \int_{0}^{T} \bar{\lambda}_{\nu} v \mathrm{~d} t \mathrm{~d} x+\mu \int_{\Omega_{\bar{u}_{\nu}}^{0}}\|v(x)\|_{L^{2}(0, T)} \mathrm{d} x .
\end{aligned}
$$

Hence, we get

$$
\int_{\Omega_{\bar{u}_{\nu}}^{0}} \int_{0}^{T} \bar{\lambda}_{\nu} v \mathrm{~d} t \mathrm{~d} x=\int_{\Omega_{\bar{u}_{\nu}}^{0}}\|v(x)\|_{L^{2}(0, T)} \mathrm{d} x .
$$

Taking into account (3.27), this leads to

$$
\bar{\lambda}_{\nu}(x, t)=\frac{v(x, t)}{\|v(x)\|_{L^{2}(0, T)}} \quad \text { if } v(x, t) \neq 0 \quad \text { a.e. in } \Omega_{\bar{u}_{\nu}}^{0} \times(0, T) .
$$


Now, we define for every $k \geq 1$ and $(x, t) \in \Omega_{\bar{u}_{\nu}} \times(0, T)$

$$
v_{k}(x, t)=\left\{\begin{array}{l}
0 \text { if } \bar{u}_{\nu}(x, t) \in\left(\alpha, \alpha+\frac{1}{k}\right) \cup\left(-\frac{1}{k}, 0\right) \cup\left(0,+\frac{1}{k}\right) \cup\left(\beta-\frac{1}{k}, \beta\right), \\
0 \text { if }\left\|\bar{u}_{\nu}(x)\right\|_{L^{2}(0, T)}<\frac{1}{k} \\
\operatorname{Proj}_{[-k,+k]}(v(x, t)) \text { otherwise, }
\end{array}\right.
$$

and for $(x, t) \in \Omega_{\bar{u}_{\nu}}^{0} \times(0, T)$ we set

$$
v_{k}(x, t)=\left\{\begin{array}{l}
0 \quad \text { if }\|v(x)\|_{L^{2}(0, T)}>k \\
v(x, t) \quad \text { otherwise. }
\end{array}\right.
$$

It is clear that $v_{k} \in \mathcal{T}_{\mathbb{K}}\left(\bar{u}_{\nu}\right)$. For every $k$ we have with (4.12)

$$
\int_{\Omega_{\bar{u}_{\nu}}^{0}} \int_{0}^{T} \bar{\lambda}_{\nu} v_{k} \mathrm{~d} t \mathrm{~d} x=\int_{\Omega_{\bar{u}_{\nu}}^{0}}\left\|v_{k}(x)\right\|_{L^{2}(0, T)} \mathrm{d} x,
$$

and also $\left(\bar{\varphi}_{\nu}+\nu \bar{u}_{\nu}+\mu \bar{\lambda}_{\nu}\right) v_{k}=0$ in $\Omega_{\bar{u}_{\nu}} \times(0, T)$. Thus, with (3.4), (3.28) and (3.32) we obtain that $v_{k} \in C_{\bar{u}_{\nu}}$. Moreover, $v_{k} \in L^{\infty}\left(\Omega_{T}\right)$ holds. Indeed, it is enough to check that $v_{k}$ is essentially bounded in $\Omega_{\bar{u}_{\nu}}^{0} \times(0, T)$. Since $\bar{\varphi}_{\nu}+\mu \bar{\lambda}_{\nu}=0$ in $\Omega_{\bar{u}_{\nu}}^{0} \times(0, T)$ and $\bar{\varphi}_{\nu} \in L^{\infty}\left(\Omega_{T}\right)$, we infer that $\bar{\lambda}_{\nu} \in L^{\infty}\left(\Omega_{\bar{u}_{\nu}}^{0} \times(0, T)\right)$. Invoking $(4.12)$ we get for $(x, t) \in \Omega_{\bar{u}_{\nu}}^{0} \times(0, T)$

$$
\begin{array}{ll}
\text { if }\|v(x)\|_{L^{2}(0, T)} \leq k & \text { then }\left|v_{k}(x, t)\right|=|v(x, t)| \leq\left|\bar{\lambda}_{\nu}(x, t)\right|\|v(x)\|_{L^{2}(0, T)} \\
& \leq\left\|\bar{\lambda}_{\nu}(x, t)\right\|_{L^{\infty}\left(\Omega_{\bar{u}_{\nu}}^{0} \times(0, T)\right)} k, \\
\text { if }\|v(x)\|_{L^{2}(0, T)}>k \quad \text { then } v_{k}(x, t)=0 .
\end{array}
$$

Thus, we have that $\alpha \leq \bar{u}+\rho v_{k} \leq \beta$ for $\rho>0$ sufficiently small. Then, we can argue as in the previous case, using again (3.24) and (3.25), with $f(t)=\bar{u}_{\nu}(x, t)$ and $g(t)=v_{k}(x, t)$, and (3.28) and (4.7) to get for $0 \leq \theta_{k, \rho}(x) \leq 1$ and $0 \leq \vartheta_{k, \rho} \leq 1$

$$
\begin{aligned}
0 \leq & J_{\nu}\left(\bar{u}_{\nu}+\rho v_{k}\right)-J_{\nu}\left(\bar{u}_{\nu}\right)=F_{\nu}\left(\bar{u}_{\nu}+\rho v_{k}\right)-F_{\nu}\left(\bar{u}_{\nu}\right) \\
& +\mu\left\{\rho \int_{\Omega_{\bar{u}_{\nu}}^{0}}\left\|v_{k}(x)\right\|_{L^{2}(0, T)} \mathrm{d} x+\int_{\Omega_{\bar{u}_{\nu}}}(\Psi(f+\rho g)-\Psi(f)) \mathrm{d} x\right\} \\
= & \rho F_{\nu}^{\prime}\left(\bar{u}_{\nu}\right) v_{k}+\frac{\rho^{2}}{2} F_{\nu}^{\prime \prime}\left(\bar{u}_{\nu}+\vartheta_{k, \rho} \rho v_{k}\right) v_{k}^{2} \\
& +\mu\left\{\rho \int_{\Omega_{\bar{u}_{\nu}}^{0}}\left\|v_{k}(x)\right\|_{L^{2}(0, T)} \mathrm{d} x+\int_{\Omega_{\bar{u}_{\nu}}}\left\{\rho \Psi^{\prime}(f) g+\frac{\rho^{2}}{2} \Psi^{\prime \prime}\left(f+\theta_{k, \rho} \rho g\right) g^{2}\right\} \mathrm{d} x\right\} \\
= & \rho\left\{F_{\nu}^{\prime}\left(\bar{u}_{\nu}\right) v_{k}+\mu j_{3}^{\prime}\left(\bar{u}_{\nu} ; v_{k}\right)\right\} \\
& +\frac{\rho^{2}}{2}\left\{F_{\nu}^{\prime \prime}\left(\bar{u}_{\nu}+\vartheta_{k, \rho} \rho v_{k}\right) v_{k}^{2}+\mu j_{3}^{\prime \prime}\left(\bar{u}_{\nu}+\theta_{k, \rho} \rho v_{k} ; v_{k}^{2}\right)\right\} \\
= & \frac{\rho^{2}}{2}\left\{F_{\nu}^{\prime \prime}\left(\bar{u}_{\nu}+\vartheta_{k, \rho} \rho v_{k}\right) v_{k}^{2}+\mu j_{3}^{\prime \prime}\left(\bar{u}_{\nu}+\theta_{k, \rho} \rho v_{k} ; v_{k}^{2}\right)\right\},
\end{aligned}
$$

hence,

$$
F_{\nu}^{\prime \prime}\left(\bar{u}_{\nu}+\vartheta_{k, \rho} \rho v_{k}\right) v_{k}^{2}+\mu j_{3}^{\prime \prime}\left(\bar{u}_{\nu}+\theta_{k, \rho} \rho v_{k} ; v_{k}^{2}\right) \geq 0 .
$$


Now, we pass to the limit as $\rho \rightarrow 0$ and $k \rightarrow \infty$. Let us denote $u_{\rho, k}=\bar{u}_{\nu}+\theta_{k, \rho} \rho v_{k}$. According to (4.7), we have

$$
j_{3}^{\prime \prime}\left(u_{\rho, k} ; v_{k}^{2}\right)=\int_{\Omega_{u_{\rho, k}}} \frac{1}{\left\|u_{\rho, k}(x)\right\|_{L^{2}(0, T)}}\left[\int_{0}^{T} v_{k}^{2}(x, t) \mathrm{d} t-\left(\int_{0}^{T} \frac{u_{\rho, k}(x, t) v_{k}(x, t)}{\left\|u_{\rho, k}(x)\right\|_{L^{2}(0, T)}} \mathrm{d} t\right)^{2}\right] \mathrm{d} x .
$$

Let us analyze the set $\Omega_{u_{\rho, k}}$. If $x \in \Omega_{\bar{u}_{\nu}}^{0}$ and $\|v(x)\|_{L^{2}(0, T)}>k$, then we get from the definition of $v_{k}$ in $\Omega_{\bar{u}_{\nu}}^{0} \times(0, T)$ that $\left\|u_{\rho, k}(x)\right\|_{L^{2}(0, T)}=0$, hence $x \notin \Omega_{u_{\rho, k}}$.

If $x \in \Omega_{\bar{u}_{\nu}}^{0} \cap \Omega_{u_{\rho, k}}$, then $\|v(x)\|_{L^{2}(0, T)} \leq k$ holds, hence $v_{k}(x, t)=v(x, t)$ and $u_{\rho, k}(x, t)=\theta_{k, \rho} \rho v(x, t)$ for a.a. $t \in(0, T)$. This implies that

$$
\int_{0}^{T} v_{k}^{2}(x, t) \mathrm{d} t-\left(\int_{0}^{T} \frac{u_{\rho, k}(x, t) v_{k}(x, t)}{\left\|u_{\rho, k}(x)\right\|_{L^{2}(0, T)}} \mathrm{d} t\right)^{2}=0 .
$$

If $x \in \Omega_{\bar{u}_{\nu}} \cap \Omega_{u_{\rho, k}}$ and $\left\|\bar{u}_{\nu}(x)\right\|_{L^{2}(0, T)}<\frac{1}{k}$, then $v_{k}(x, t)=0$ and the integrand in $j_{3}^{\prime \prime}\left(u_{\rho, k} ; v_{k}^{2}\right)$ vanishes again. Therefore, we have that

$$
\begin{aligned}
& j_{3}^{\prime \prime}\left(u_{\rho, k} ; v_{k}^{2}\right) \\
& =\int_{\Omega_{\rho, k}} \frac{1}{\left\|u_{\rho, k}(x)\right\|_{L^{2}(0, T)}}\left[\int_{0}^{T} v_{k}^{2}(x, t) \mathrm{d} t-\left(\int_{0}^{T} \frac{u_{\rho, k}(x, t) v_{k}(x, t)}{\left\|u_{\rho, k}(x)\right\|_{L^{2}(0, T)}} \mathrm{d} t\right)^{2}\right] \mathrm{d} x
\end{aligned}
$$

with

$$
\Omega_{\rho, k}=\left\{x \in \Omega_{u_{\rho, k}}:\left\|\bar{u}_{\nu}(x)\right\|_{L^{2}(0, T)} \geq \frac{1}{k}\right\} .
$$

Using that $\left|v_{k}(x, t)\right| \leq k$ in $\Omega_{\bar{u}_{\nu}} \times(0, T)$, we obtain for every $0<\rho<\frac{1}{2 k^{2} \sqrt{T}}$ and a.a. $x \in \Omega_{\rho, k}$

$$
\left\|u_{\rho, k}(x)\right\|_{L^{2}(0, T)} \geq\left\|\bar{u}_{\nu}(x)\right\|_{L^{2}(0, T)}-\theta_{k, \rho} \rho\left\|v_{k}\right\|_{L^{2}(0, T)} \geq \frac{1}{k}-\frac{1}{2 k^{2} \sqrt{T}} k \sqrt{T}=\frac{1}{2 k} .
$$

Then, for $k$ fixed, we easily get that $\lim _{\rho \rightarrow 0} j_{3}^{\prime \prime}\left(u_{\rho, k} ; v_{k}^{2}\right)=j_{3}^{\prime \prime}\left(\bar{u}_{\nu} ; v_{k}^{2}\right)$, and hence passing to the limit in $(4.13)$ as $\rho \rightarrow 0$ we deduce that $J_{\nu}^{\prime \prime}\left(\bar{u}_{\nu} ; v_{k}^{2}\right) \geq 0$. Now, we can pass to the limit as $k \rightarrow \infty$ using the Lebesgue dominated convergence theorem and taking into account that, by construction, $v_{k}(x, t) \rightarrow v(x, t)$ a.e., $(4.11)$ and the fact that

$$
\begin{aligned}
0 & \leq \frac{1}{\left\|\bar{u}_{\nu}(x)\right\|_{L^{2}(0, T)}}\left[\int_{0}^{T} v_{k}^{2}(x, t) \mathrm{d} t-\left(\int_{0}^{T} \frac{\bar{u}_{\nu}(x, t) v_{k}(x, t)}{\left\|\bar{u}_{\nu}(x)\right\|_{L^{2}(0, T)}} \mathrm{d} t\right)^{2}\right] \\
& \leq \frac{\left\|v_{k}(x)\right\|_{L^{2}(0, T)}^{2}}{\left\|\bar{u}_{\nu}(x)\right\|_{L^{2}(0, T)}} \leq \frac{\|v(x)\|_{L^{2}(0, T)}^{2}}{\left\|\bar{u}_{\nu}(x)\right\|_{L^{2}(0, T)}}
\end{aligned}
$$

Note that the latter term (as a function of $x$ ) belongs to $L^{1}\left(\Omega_{\bar{u}_{\nu}}\right)$ and provides an integrable majorant. Thus, we have that $J_{\nu}^{\prime \prime}\left(\bar{u}_{\nu} ; v^{2}\right) \geq 0$ for every $v \in C_{\bar{u}_{\nu}}$ satisfying (4.11). We finish the proof by removing the assumption (4.11). For an arbitrary element $v \in C_{\bar{u}_{\nu}}$, we define

$$
v_{k}(x, t)=\left\{\begin{array}{cl}
0 \quad \text { if } 0<\left\|\bar{u}_{\nu}(x)\right\|_{L^{2}(0, T)}<\frac{1}{k} \\
v(x, t) \text { otherwise. }
\end{array}\right.
$$

As above, we can prove that $v_{k} \in C_{\bar{u}_{\nu}}$. Moreover, $v_{k}$ satisfies (4.11). Hence, $J_{\nu}^{\prime \prime}\left(\bar{u}_{\nu} ; v_{k}^{2}\right) \geq 0$. We have that the integrands in $j_{3}^{\prime \prime}\left(\bar{u}_{\nu} ; v_{k}^{2}\right)$ are nonnegative and they form an increasing sequence in $k$. Therefore, we can apply the Lebesgue monotone convergence theorem to pass to the limit and deduce that $j_{3}^{\prime \prime}\left(\bar{u}_{\nu} ; v^{2}\right)=\lim _{k \rightarrow \infty} j_{3}^{\prime \prime}\left(\bar{u}_{\nu} ; v_{k}^{2}\right)$, and hence $J_{\nu}^{\prime \prime}\left(\bar{u}_{\nu} ; v^{2}\right)=\lim _{k \rightarrow \infty} J_{\nu}^{\prime \prime}\left(\bar{u}_{\nu} ; v_{k}^{2}\right) \geq 0$. 


\section{SECOND-ORDER SUFFICIENT Optimality CONDITIONS}

Hereafter $\bar{u}_{\nu}$ will denote an element of $\mathbb{K}$ that along with $\left(\bar{y}_{\nu}, \bar{\varphi}_{\nu}, \bar{\lambda}_{\nu}\right) \in Y \times \Phi \times \partial j\left(\bar{u}_{\nu}\right)$ satisfies the optimality system (3.2)-(3.4). Associated to $\bar{u}_{\nu}$, we define the extended cones of critical directions for $\tau \geq 0$

$$
C_{\bar{u}_{\nu}}^{\tau}=\left\{v \in \mathcal{T}_{\mathbb{K}}\left(\bar{u}_{\nu}\right): F_{\nu}^{\prime}\left(\bar{u}_{\nu}\right) v+\mu j^{\prime}\left(\bar{u}_{\nu} ; v\right) \leq \tau\|v\|_{L^{2}\left(\Omega_{T}\right)}\right\} .
$$

Recall that $F_{\nu}^{\prime}\left(\bar{u}_{\nu}\right) v+\mu j^{\prime}\left(\bar{u}_{\nu} ; v\right) \geq 0$, due to Lemma 4.2. It is obvious that $C_{\bar{u}_{\nu}}^{0}$ coincides with the cone $C_{\bar{u}_{\nu}}$ defined in (4.1), and $C_{\bar{u}_{\nu}} \subset C_{\bar{u}_{\nu}}^{\tau}$ for every $\tau>0$.

\subsection{Problem $\left(\mathrm{P}_{\nu}^{1}\right)$}

For this problem we recall that, by definition, $J_{\nu}^{\prime \prime}\left(\bar{u}_{\nu} ; v^{2}\right)=F_{\nu}^{\prime \prime}\left(\bar{u}_{\nu}\right) v^{2}$; see (4.5). Therefore we can formulate the second-order sufficient condition in terms of $F_{\nu}^{\prime \prime}$. We will distinguish the cases $\nu>0$ and $\nu=0$. For $\nu>0$ there are different equivalent ways of formulating the second-order sufficient optimality conditions.

Theorem 5.1. Let us assume that $\nu>0$. Then the following statements are equivalent

$$
\begin{aligned}
& F_{\nu}^{\prime \prime}\left(\bar{u}_{\nu}\right) v^{2}>0 \forall v \in C_{\bar{u}_{\nu}} \backslash\{0\} \\
& \exists \delta>0 \text { and } \tau>0 \text { such that } F_{\nu}^{\prime \prime}\left(\bar{u}_{\nu}\right) v^{2} \geq \delta\|v\|_{L^{2}\left(\Omega_{T}\right)}^{2} \quad \forall v \in C_{\bar{u}_{\nu}}^{\tau}, \\
& \exists \delta>0 \text { and } \tau>0 \text { such that } F_{\nu}^{\prime \prime}\left(\bar{u}_{\nu}\right) v^{2} \geq \delta\left\|z_{v}\right\|_{L^{2}\left(\Omega_{T}\right)}^{2} \forall v \in C_{\bar{u}_{\nu}}^{\tau} .
\end{aligned}
$$

where $z_{v}=G^{\prime}\left(\bar{u}_{\nu}\right) v$ is the solution of the linearized parabolic equation (2.7) corresponding to $y_{u}=\bar{y}_{\nu}$.

The implications $(5.3) \Rightarrow(5.4) \Rightarrow(5.2)$ are obvious. Indeed, from (2.7) we know that $z_{u, v}=G^{\prime}(u) v$ satisfies

$$
\left\|z_{u, v}\right\|_{L^{2}\left(\Omega_{T}\right)} \leq C_{\mathbb{K}}\|v\|_{L^{2}\left(\Omega_{T}\right)} \quad \forall u \in \mathbb{K} \text { and } \forall v \in L^{2}\left(\Omega_{T}\right)
$$

for some $C_{\mathbb{K}}>0$. Therefore, it is immediate that (5.3) implies (5.4) with the same $\tau$ and replacing $\delta$ in (5.4) by $\delta / C_{\mathbb{K}}^{2}$. The only delicate part is the proof that (5.2) implies (5.3). To this end, it is enough to proceed as in the elliptic case in ([5], Thm. 3.8).

$$
\left\|v_{k}\right\|_{L^{2}\left(\Omega_{T}\right)}=1, \quad v_{k} \rightarrow v \text { in } L^{2}\left(\Omega_{T}\right) \quad \text { and } \quad F_{\nu}^{\prime \prime}\left(\bar{u}_{\nu}\right) v_{k}^{2}<\frac{1}{k} .
$$

Before establishing the theorem on the second-order sufficient conditions, we need to prove a technical lemma.

Lemma 5.2. For every $\rho>0$ there exists $\varepsilon>0$ such that

$$
\left|\left[F_{\nu}^{\prime \prime}(u)-F_{\nu}^{\prime \prime}\left(\bar{u}_{\nu}\right)\right] v^{2}\right| \leq \rho\left\|z_{v}\right\|_{L^{2}\left(\Omega_{T}\right)}^{2} \forall v \in L^{2}\left(\Omega_{T}\right) \text { and } \forall u \in \mathbb{K} \cap B_{\varepsilon}\left(\bar{u}_{\nu}\right),
$$

where $z_{v}=G^{\prime}\left(\bar{u}_{\nu}\right) v$ and $B_{\varepsilon}\left(\bar{u}_{\nu}\right)$ denotes the ball of $L^{2}\left(\Omega_{T}\right)$ centered at $\bar{u}_{\nu}$ and with radius $\varepsilon$.

Proof. First, we get from (1.1) and the boundedness of $\mathbb{K}$ in $L^{\infty}\left(\Omega_{T}\right)$

$$
\exists M_{\mathbb{K}} \text { such that }\left\|y_{u}\right\|_{L^{\infty}\left(\Omega_{T}\right)} \leq M_{\mathbb{K}} \forall u \in \mathbb{K} .
$$

Now, for every $u \in \mathbb{K}$, subtracting the equations satisfied by $y_{u}$ and $\bar{y}_{\nu}$, and using the mean value theorem we obtain

$$
\left\{\begin{aligned}
\frac{\partial\left(y_{u}-\bar{y}_{\nu}\right)}{\partial t}+A\left(y_{u}-\bar{y}_{\nu}\right)+\frac{\partial a}{\partial y}\left(x, t, \bar{y}_{\nu}+\theta\left(y_{u}-\bar{y}_{\nu}\right)\right)\left(y_{u}-\bar{y}_{\nu}\right) & =u-\bar{u}_{\nu} & & \text { in } \Omega_{T}, \\
y_{u}-\bar{y}_{\nu} & =0 & & \text { on } \Sigma_{T}, \\
\left(y_{u}-\bar{y}_{\nu}\right)(0) & =0 & & \text { in } \Omega .
\end{aligned}\right.
$$


From here, it follows

$$
\left\|y_{u}-\bar{y}_{\nu}\right\|_{L^{\infty}\left(\Omega_{T}\right)} \leq C\left\|u-\bar{u}_{\nu}\right\|_{L^{3}\left(\Omega_{T}\right)} \leq C_{1}\left\|u-\bar{u}_{\nu}\right\|_{L^{2}\left(\Omega_{T}\right)}^{2 / 3} \forall u \in \mathbb{K} .
$$

We proceed with the adjoint states $\varphi_{u}$ and $\bar{\varphi}_{\nu}$ in a similar way

$$
\left\{\begin{array}{cc}
-\frac{\partial\left(\varphi_{u}-\bar{\varphi}_{\nu}\right)}{\partial t}+A^{*}\left(\varphi_{u}-\bar{\varphi}_{\nu}\right)+\frac{\partial a}{\partial y}\left(x, t, \bar{y}_{\nu}\right)\left(\varphi_{u}-\bar{\varphi}_{\nu}\right) & \\
=\frac{\partial L}{\partial y}\left(x, t, y_{u}\right)-\frac{\partial L}{\partial y}\left(x, t, \bar{y}_{\nu}\right)+\left[\frac{\partial a}{\partial y}\left(x, t, \bar{y}_{\nu}\right)-\frac{\partial a}{\partial y}\left(x, t, y_{u}\right)\right] \varphi_{u} & \text { in } \Omega_{T}, \\
\left(\varphi_{u}-\bar{\varphi}_{\nu}\right)=0 & \text { on } \Sigma_{T}, \\
\left(\varphi_{u}-\bar{\varphi}_{\nu}\right)(T)=0 & \text { in } \Omega .
\end{array}\right.
$$

From (5.8) and (5.9) along with the assumptions (2.3) and (2.5) we obtain

$$
\left\|\varphi_{u}-\bar{\varphi}_{\nu}\right\|_{L^{\infty}\left(\Omega_{T}\right)} \leq C\left\|y_{u}-\bar{y}_{\nu}\right\|_{L^{\infty}\left(\Omega_{T}\right)} \leq C_{2}\left\|u-\bar{u}_{\nu}\right\|_{L^{2}\left(\Omega_{T}\right)}^{2 / 3} \forall u \in \mathbb{K} .
$$

Let us denote $z_{u, v}=G^{\prime}(u) v$ and $z_{v}=G^{\prime}\left(\bar{u}_{\nu}\right) v$. We put $z=z_{u, v}-z_{v}$. Subtracting the equations satisfied by $z_{u, v}$ and $z_{v}$ we get

$$
\left\{\begin{aligned}
\frac{\partial z}{\partial t}+A z+\frac{\partial a}{\partial y}\left(x, t, y_{u}\right) z & =\left[\frac{\partial a}{\partial y}\left(x, t, \bar{y}_{\nu}\right)-\frac{\partial a}{\partial y}\left(x, t, y_{u}\right)\right] z_{v} & & \text { in } \Omega_{T}, \\
z & =0 & & \text { on } \Sigma_{T}, \\
z(0) & =0 & & \text { in } \Omega .
\end{aligned}\right.
$$

From the mean value theorem and (2.3) we deduce

$$
\left\|z_{u, v}-z_{v}\right\|_{L^{2}\left(\Omega_{T}\right)} \leq C\left\|y_{u}-\bar{y}_{\nu}\right\|_{L^{\infty}\left(\Omega_{T}\right)}\left\|z_{v}\right\|_{L^{2}\left(\Omega_{T}\right)} \quad \forall u \in \mathbb{K} \text { and } \forall v \in L^{2}\left(\Omega_{T}\right) .
$$

Hence, we also have

$$
\left\|z_{u, v}\right\|_{L^{2}\left(\Omega_{T}\right)} \leq\left(1+C\left\|y_{u}-\bar{y}_{\nu}\right\|_{L^{\infty}\left(\Omega_{T}\right)}\right)\left\|z_{v}\right\|_{L^{2}\left(\Omega_{T}\right)} \forall u \in \mathbb{K} \text { and } \forall v \in L^{2}\left(\Omega_{T}\right) .
$$

From the expression (2.10) we infer

$$
\begin{aligned}
\left|\left[F_{\nu}^{\prime \prime}(u)-F_{\nu}^{\prime \prime}\left(\bar{u}_{\nu}\right)\right] v^{2}\right| \leq & \left|\int_{\Omega_{T}}\left(\frac{\partial^{2} L}{\partial^{2} y}\left(x, t, y_{u}\right)-\varphi_{u} \frac{\partial^{2} a}{\partial^{2} y}\left(x, t, y_{u}\right)\right)\left(z_{u, v}^{2}-z_{v}^{2}\right) \mathrm{d} x \mathrm{~d} t\right| \\
& +\int_{\Omega_{T}}\left|\frac{\partial^{2} L}{\partial^{2} y}\left(x, t, y_{u}\right)-\frac{\partial^{2} L}{\partial^{2} y}\left(x, t, \bar{y}_{\nu}\right)\right| z_{v}^{2} \mathrm{~d} x \mathrm{~d} t \\
& +\int_{\Omega_{T}}\left|\bar{\varphi}_{\nu}\right|\left|\frac{\partial^{2} a}{\partial^{2} y}\left(x, t, y_{u}\right)-\frac{\partial^{2} a}{\partial^{2} y}\left(x, t, \bar{y}_{\nu}\right)\right| z_{v}^{2} \mathrm{~d} x \mathrm{~d} t \\
& +\int_{\Omega_{T}}\left|\varphi_{u}-\bar{\varphi}_{\nu}\right|\left|\frac{\partial^{2} a}{\partial^{2} y}\left(x, t, y_{u}\right)\right| z_{v}^{2} \mathrm{~d} x \mathrm{~d} t=I_{1}+I_{2}+I_{3}+I_{4} .
\end{aligned}
$$

Let us estimate these four terms. First, (2.3), (2.5), (5.8), (5.11) and (5.12) imply

$$
\begin{aligned}
I_{1} & \leq\left\|\frac{\partial^{2} L}{\partial^{2} y}\left(x, t, y_{u}\right)-\varphi_{u} \frac{\partial^{2} a}{\partial^{2} y}\left(x, t, y_{u}\right)\right\|_{L^{\infty}\left(\Omega_{T}\right)}\left\|z_{u, v}-z_{v}\right\|_{L^{2}\left(\Omega_{T}\right)}\left\|z_{u, v}+z_{v}\right\|_{L^{2}\left(\Omega_{T}\right)} \\
& \leq C_{3}\left\|y_{u}-\bar{y}_{\nu}\right\|_{L^{\infty}\left(\Omega_{T}\right)}\left\|z_{v}\right\|_{L^{2}\left(\Omega_{T}\right)}^{2} .
\end{aligned}
$$


Using (5.9) and taking $0<\varepsilon_{1} \leq\left(\frac{\rho}{4 C_{1} C_{3}}\right)^{3 / 2}$, we deduce from the above inequality

$$
I_{1} \leq \frac{\rho}{4}\left\|z_{v}\right\|_{L^{2}\left(\Omega_{T}\right)}^{2} \quad \text { if } \quad\left\|u-\bar{u}_{\nu}\right\|_{L^{2}\left(\Omega_{T}\right)} \leq \varepsilon_{1} .
$$

Using again (5.9) along with Assumption (2.6), we infer the existence of $\varepsilon_{2}>0$ such that $I_{2}$ satisfies the above inequality for $\left\|u-\bar{u}_{\nu}\right\|_{L^{2}\left(\Omega_{T}\right)} \leq \varepsilon_{2}$. Analogously, using (5.9), (2.4) and the fact that $\bar{\varphi}_{\nu} \in L^{\infty}\left(\Omega_{T}\right)$, we deduce the same estimate for $I_{3}$ for some $\varepsilon_{3}>0$. Finally, from (5.8), (5.10) and (2.3) we get

$$
\begin{aligned}
I_{4} & \leq\left\|\varphi_{u}-\bar{\varphi}_{\nu}\right\|_{L^{\infty}\left(\Omega_{T}\right)}\left\|\frac{\partial^{2} a}{\partial^{2} y}\left(x, t, y_{u}\right)\right\|_{L^{\infty}\left(\Omega_{T}\right)}\left\|z_{v}\right\|_{L^{2}\left(\Omega_{T}\right)}^{2} \\
& \leq C_{4}\left\|u-\bar{u}_{\nu}\right\|_{L^{2}\left(\Omega_{T}\right)}^{2 / 3}\left\|z_{v}\right\|_{L^{2}\left(\Omega_{T}\right)}^{2} .
\end{aligned}
$$

Now, taking $0<\varepsilon_{4} \leq\left(\frac{\rho}{4 C_{4}}\right)^{3 / 2}$, we obtain the corresponding estimate for $I_{4}$. Adding the estimates for $I_{i}$, we conclude (5.7) for $\varepsilon=\min _{1 \leq i \leq 4} \varepsilon_{i}$.

Theorem 5.3. Let $\nu>0$ and assume that $\bar{u}_{\nu}$ satisfies (5.3). Then there exists $\varepsilon>0$ such that

$$
J_{\nu}\left(\bar{u}_{\nu}\right)+\frac{\delta}{4}\left\|u-\bar{u}_{\nu}\right\|_{L^{2}\left(\Omega_{T}\right)}^{2} \leq J_{\nu}(u) \quad \forall u \in \mathbb{K} \cap B_{\varepsilon}\left(\bar{u}_{\nu}\right),
$$

where $B_{\varepsilon}\left(\bar{u}_{\nu}\right)$ denotes the ball of $L^{2}\left(\Omega_{T}\right)$ centered at $\bar{u}_{\nu}$ and with radius $\varepsilon$.

Proof. Let us define $\varepsilon$. From (2.3), (2.5), (5.8), (5.5), and the expression (5.10), we obtain $\forall u \in \mathbb{K}$ and $\forall v \in$ $L^{2}\left(\Omega_{T}\right)$

$$
\left|F^{\prime \prime}(u) v^{2}\right| \leq C_{0}\left\|z_{u, v}\right\|_{L^{2}\left(\Omega_{T}\right)^{2}}^{2}+\nu\|v\|_{L^{2}\left(\Omega_{T}\right)}^{2} \leq\left(C_{0} C_{\mathbb{K}}^{2}+\nu\right)\|v\|_{L^{2}\left(\Omega_{T}\right)}^{2} .
$$

We take $\varepsilon_{1}$ satisfying

$$
0<\varepsilon_{1} \leq \frac{4 \tau}{\delta+2\left(C_{0} C_{\mathbb{K}}^{2}+\nu\right)} .
$$

On the other hand, applying Lemma 5.2 we obtain $\varepsilon_{2}>0$ such that $\forall u \in \mathbb{K} \cap B_{\varepsilon_{2}}\left(\bar{u}_{\nu}\right)$ and $\forall v \in L^{2}\left(\Omega_{T}\right)$

$$
\left|\left[F_{\nu}^{\prime \prime}(u)-F_{\nu}^{\prime \prime}\left(\bar{u}_{\nu}\right)\right] v^{2}\right| \leq \frac{\delta}{2 C_{\mathbb{K}}^{2}}\left\|z_{v}\right\|_{L^{2}\left(\Omega_{T}\right)}^{2} \leq \frac{\delta}{2}\|v\|_{L^{2}\left(\Omega_{T}\right)}^{2} .
$$

Finally, we set $\varepsilon=\min \left\{\varepsilon_{1}, \varepsilon_{2}\right\}$. Let us take $u \in \mathbb{K} \cap B_{\varepsilon}\left(\bar{u}_{\nu}\right)$. We will distinguish two cases.

Case I: $u-\bar{u}_{\nu} \notin C_{\bar{u}_{\nu}}^{\tau}$. - Since $u-\bar{u}_{\nu} \in \mathcal{T}_{\mathbb{K}}\left(\bar{u}_{\nu}\right)$, then from the definition (5.1) it follows

$$
F_{\nu}^{\prime}\left(\bar{u}_{\nu}\right)\left(u-\bar{u}_{\nu}\right)+\mu j_{1}^{\prime}\left(\bar{u}_{\nu} ; u-\bar{u}_{\nu}\right)>\tau\left\|u-\bar{u}_{\nu}\right\|_{L^{2}\left(\Omega_{T}\right)} .
$$

Hence, making a Taylor expansion of $F_{\nu}(u)$ around $\bar{u}_{\nu}$, using (4.4), (5.14), and the above inequality we get

$$
\begin{aligned}
J_{\nu}(u)-J_{\nu}\left(\bar{u}_{\nu}\right) & \geq F_{\nu}^{\prime}\left(\bar{u}_{\nu}\right)\left(u-\bar{u}_{\nu}\right)+\mu j_{1}^{\prime}\left(\bar{u}_{\nu} ; u-\bar{u}_{\nu}\right)+\frac{1}{2} F_{\nu}^{\prime \prime}\left(\bar{u}_{\nu}+\theta\left(u-\bar{u}_{\nu}\right)\right)\left(u-\bar{u}_{\nu}\right)^{2} \\
& \geq \tau\left\|u-\bar{u}_{\nu}\right\|_{L^{2}\left(\Omega_{T}\right)}-\frac{1}{2}\left(C_{0} C_{\mathbb{K}}^{2}+\nu\right)\left\|u-\bar{u}_{\nu}\right\|_{L^{2}\left(\Omega_{T}\right)}^{2} \\
& \geq\left[\frac{\tau}{\varepsilon}-\frac{1}{2}\left(C_{0} C_{\mathbb{K}}^{2}+\nu\right)\right]\left\|u-\bar{u}_{\nu}\right\|_{L^{2}\left(\Omega_{T}\right)}^{2} .
\end{aligned}
$$

From the definition of $\varepsilon$ and $\varepsilon_{1}$, we deduce (5.13) from the above inequality. 
Case II: $u-\bar{u}_{\nu} \in C_{\bar{u}_{\nu}}^{\tau}$ - - Now, from (4.2), (5.3), and (5.15) we infer

$$
\begin{aligned}
J_{\nu}(u)-J_{\nu}\left(\bar{u}_{\nu}\right) & \geq F_{\nu}^{\prime}\left(\bar{u}_{\nu}\right)\left(u-\bar{u}_{\nu}\right)+\mu j_{1}^{\prime}\left(\bar{u}_{\nu} ; u-\bar{u}_{\nu}\right)+\frac{1}{2} F_{\nu}^{\prime \prime}\left(\bar{u}_{\nu}+\theta\left(u-\bar{u}_{\nu}\right)\right)\left(u-\bar{u}_{\nu}\right)^{2} \\
& \geq \frac{1}{2} F_{\nu}^{\prime \prime}\left(\bar{u}_{\nu}\right)\left(u-\bar{u}_{\nu}\right)^{2}+\frac{1}{2}\left[F_{\nu}^{\prime \prime}\left(\bar{u}_{\nu}+\theta\left(u-\bar{u}_{\nu}\right)\right)-F_{\nu}^{\prime \prime}\left(\bar{u}_{\nu}\right)\right]\left(u-\bar{u}_{\nu}\right)^{2} \\
& \geq \frac{\delta}{2}\left\|u-\bar{u}_{\nu}\right\|_{L^{2}\left(\Omega_{T}\right)}^{2}-\frac{\delta}{4}\left\|u-\bar{u}_{\nu}\right\|_{L^{2}\left(\Omega_{T}\right)}^{2}=\frac{\delta}{4}\left\|u-\bar{u}_{\nu}\right\|_{L^{2}\left(\Omega_{T}\right)}^{2} .
\end{aligned}
$$

Next we consider the case $\nu=0$. Under this assumption the relations (5.2)-(5.5) are not equivalent. It is known that (5.2) is not a sufficient second-order condition for local optimality, in general; see the example by Dunn [7]. On the other hand, (5.3) is never fulfilled for $\nu=0$. Indeed, the reader is referred to [3] for the proof of this statement in the case of an elliptic state equation, which can be reproduced in the parabolic case just replacing $x$ by $(x, t)$. Finally, we prove that the condition (5.4) is sufficient for the local optimality of $\bar{u}_{\nu}$. However, the conclusion (5.13) does not hold. A weaker consequence is deduced from (5.4) for $\nu=0$.

Theorem 5.4. Let $\nu=0$ and assume that $\bar{u}_{\nu}$ satisfies (5.4). Then there exists $\varepsilon>0$ such that

$$
J_{\nu}\left(\bar{u}_{\nu}\right)+\frac{\delta}{4}\left\|z_{u-\bar{u}_{\nu}}\right\|_{L^{2}\left(\Omega_{T}\right)}^{2} \leq J_{\nu}(u) \quad \forall u \in \mathbb{K} \cap B_{\varepsilon}\left(\bar{u}_{\nu}\right),
$$

where $z_{u-\bar{u}_{\nu}}=G^{\prime}\left(\bar{u}_{\nu}\right)\left(u-\bar{u}_{\nu}\right)$ and $B_{\varepsilon}\left(\bar{u}_{\nu}\right)$ denotes the ball of $L^{2}\left(\Omega_{T}\right)$ centered at $\bar{u}_{\nu}$ and with radius $\varepsilon$.

Proof. The proof follows the same steps as the preceding one, with minor modifications. Let us point out the changes. First, we take

$$
0<\varepsilon_{1} \leq \frac{4 \tau}{C_{\mathbb{K}}^{2}\left(\delta+2 C_{0}\right)},
$$

where $C_{0}$ was introduced in (5.14). Second, using again Lemma 5.2 we obtain $\varepsilon_{2}>0$ such that $\forall u \in \mathbb{K} \cap B_{\varepsilon_{2}}\left(\bar{u}_{\nu}\right)$ and $\forall v \in L^{2}\left(\Omega_{T}\right)$

$$
\left|\left[F_{\nu}^{\prime \prime}(u)-F_{\nu}^{\prime \prime}\left(\bar{u}_{\nu}\right)\right] v^{2}\right| \leq \frac{\delta}{2}\left\|z_{v}\right\|_{L^{2}\left(\Omega_{T}\right)}^{2} .
$$

Finally, we set $\varepsilon=\min \left\{\varepsilon_{1}, \varepsilon_{2}\right\}$. Now, if $u-\bar{u}_{\nu} \notin C_{\bar{u}_{\nu}}^{\tau}$, then we argue as in the proof of Theorem 5.3, using this time the first inequality of (5.14) and later (5.5) to deduce

$$
\begin{aligned}
J_{\nu}(u)-J_{\nu}\left(\bar{u}_{\nu}\right) & \geq F_{\nu}^{\prime}\left(\bar{u}_{\nu}\right)\left(u-\bar{u}_{\nu}\right)+\mu j_{1}^{\prime}\left(\bar{u}_{\nu} ; u-\bar{u}_{\nu}\right)+\frac{1}{2} F_{\nu}^{\prime \prime}\left(\bar{u}_{\nu}+\theta\left(u-\bar{u}_{\nu}\right)\right)\left(u-\bar{u}_{\nu}\right)^{2} \\
& \geq \tau\left\|u-\bar{u}_{\nu}\right\|_{L^{2}\left(\Omega_{T}\right)}-\frac{1}{2} C_{0}\left\|z_{u-\bar{u}_{\nu}}\right\|_{L^{2}\left(\Omega_{T}\right)}^{2} \\
& \geq \frac{\tau}{\varepsilon}\left\|u-\bar{u}_{\nu}\right\|_{L^{2}\left(\Omega_{T}\right)}^{2}-\frac{1}{2} C_{0}\left\|z_{u-\bar{u}_{\nu}}\right\|_{L^{2}\left(\Omega_{T}\right)}^{2} \\
& \geq\left(\frac{\tau}{\varepsilon C_{\mathbb{K}}^{2}}-\frac{1}{2} C_{0}\right)\left\|z_{u-\bar{u}_{\nu}}\right\|_{L^{2}\left(\Omega_{T}\right)}^{2} \geq \frac{\delta}{4}\left\|z_{u-\bar{u}_{\nu}}\right\|_{L^{2}\left(\Omega_{T}\right)}^{2} .
\end{aligned}
$$

If $u-\bar{u}_{\nu} \in C_{\bar{u}_{\nu}}^{\tau}$ we proceed exactly as in the proof of Theorem 5.3 using (5.17) and (5.4) instead of (5.15) and (5.3).

\subsection{Problem $\left(\mathrm{P}_{\nu}^{2}\right)$}

For the problem $\left(\mathrm{P}_{\nu}^{2}\right)$ we have that $J_{\nu}^{\prime \prime}\left(\bar{u}_{\nu} ; v^{2}\right)=F_{\nu}^{\prime \prime}\left(\bar{u}_{\nu}\right) v^{2}+\mu j_{2}^{\prime \prime}\left(\bar{u}_{\nu} ; v^{2}\right)$, where $j_{2}^{\prime \prime}\left(\bar{u}_{\nu} ; v^{2}\right)$ is defined by $(4.6)$. Though the term $j_{2}^{\prime \prime}\left(\bar{u}_{\nu} ; v^{2}\right)$ can help to the coercivity of the second derivative $J_{\nu}^{\prime \prime}\left(\bar{u}_{\nu} ; v^{2}\right)$, it makes the analysis of the second-order conditions technically more complicated, as we will see in the next theorem. 
Theorem 5.5. Let us assume that $\nu>0$. Then the following statements are equivalent

$$
\begin{aligned}
& J_{\nu}^{\prime \prime}\left(\bar{u}_{\nu} ; v^{2}\right)>0 \forall v \in C_{\bar{u}_{\nu}} \backslash\{0\} \\
& \exists \delta>0 \text { and } \tau>0 \text { such that } J_{\nu}^{\prime \prime}\left(\bar{u}_{\nu} ; v^{2}\right) \geq \delta\|v\|_{L^{2}\left(\Omega_{T}\right)}^{2} \forall v \in C_{\bar{u}_{\nu}}^{\tau}, \\
& \exists \delta>0 \text { and } \tau>0 \text { such that } J_{\nu}^{\prime \prime}\left(\bar{u}_{\nu} ; v^{2}\right) \geq \delta\left\|z_{v}\right\|_{L^{2}\left(\Omega_{T}\right)}^{2} \forall v \in C_{\bar{u}_{\nu}}^{\tau},
\end{aligned}
$$

where $z_{v}=G^{\prime}(\bar{u}) v$ is the solution of $(2.7)$ corresponding to $y_{u}=\bar{y}_{\nu}$.

Proof. Analogously to the proof of Theorem 5.1, we have that (5.19) $\Rightarrow$ (5.20) $\Rightarrow$ (5.18), with the same $\tau$ in (5.10) and (5.20), and taking $\delta>0$ in (5.19) and $\frac{\delta}{C_{\mathbb{K}}^{2}}$ in (5.20). The rest is dedicated to the proof of the implication $(5.18) \Rightarrow(5.19)$. We proceed by contradiction and assume that for every integer $k>0$ there exists an element $v_{k} \in C_{\bar{u}_{\nu}}^{1 / k}$ such that $J_{\nu}^{\prime \prime}\left(\bar{u}_{\nu}\right) v_{k}^{2}<\frac{1}{k}\left\|v_{k}\right\|_{L^{2}\left(\Omega_{T}\right)}^{2}$. Dividing $v_{k}$ by its $L^{2}\left(\Omega_{T}\right)$-norm and taking a subsequence, if necessary, we get for some $v \in L^{2}\left(\Omega_{T}\right)$

$$
\left\|v_{k}\right\|_{L^{2}\left(\Omega_{T}\right)}=1, \quad v_{k} \rightarrow v \text { in } L^{2}\left(\Omega_{T}\right) \quad \text { and } \quad J_{\nu}^{\prime \prime}\left(\bar{u}_{\nu} ; v_{k}^{2}\right)<\frac{1}{k},
$$

with $v \in C_{\bar{u}_{\nu}}$. Moreover, using (4.2) and (5.21), and the fact that $v_{k} \in C_{\bar{u}_{\nu}}^{1 / k}$ we obtain

$$
\begin{aligned}
0 & \leq F_{\nu}^{\prime}\left(\bar{u}_{\nu}\right) v+\mu j_{2}^{\prime}\left(\bar{u}_{\nu} ; v\right) \leq \liminf _{k \rightarrow \infty}\left\{F^{\prime}\left(\bar{u}_{\nu}\right) v_{k}+\mu j_{2}^{\prime}\left(\bar{u}_{\nu} ; v_{k}\right)\right\} \\
& \leq \limsup _{k \rightarrow \infty}\left\{F^{\prime}\left(\bar{u}_{\nu}\right) v_{k}+\mu j_{2}^{\prime}\left(\bar{u}_{\nu} ; v_{k}\right)\right\}=0 .
\end{aligned}
$$

Since $F_{\nu}^{\prime}\left(\bar{u}_{\nu}\right) v_{k} \rightarrow F_{\nu}^{\prime}\left(\bar{u}_{\nu}\right) v$, we deduce that $\lim _{k \rightarrow \infty} j_{2}^{\prime}\left(\bar{u}_{\nu} ; v_{k}\right)=j_{2}^{\prime}\left(\bar{u}_{\nu} ; v\right)$. In case $\bar{u}_{\nu} \neq 0$, we can apply Lemma 5.6 below, and obtain that the sequence of functions $t \mapsto j_{\Omega}^{\prime}\left(\bar{u}_{\nu}(t) ; v_{k}(t)\right)$ converges weakly in $L^{2}(0, T)$ to $t \mapsto j_{\Omega}^{\prime}\left(\bar{u}_{\nu}(t) ; v(t)\right)$. From the expression (4.6) for $j_{2}^{\prime \prime}$ and these two convergence properties, we get that $j_{2}^{\prime \prime}\left(\bar{u}_{\nu} ; v^{2}\right) \leq \liminf _{k \rightarrow \infty} j_{2}^{\prime \prime}\left(\bar{u}_{\nu} ; v_{k}^{2}\right)$. In case $\bar{u}_{\nu}=0$ this also holds true, since $j_{2}^{\prime \prime}(0 ; \cdot) \equiv 0$ by (4.6). Hence, $(5.21)$ leads to

$$
J_{\nu}^{\prime \prime}\left(\bar{u}_{\nu} ; v^{2}\right) \leq \liminf _{k \rightarrow \infty} J_{\nu}^{\prime \prime}\left(\bar{u}_{\nu} ; v_{k}^{2}\right) \leq 0 .
$$

This inequality and (5.18) imply that $v=0$. Then, we deduce as in the proof of Theorem 5.1 that $\nu=0$, which contradicts our assumption.

Lemma 5.6. Let $\left\{v_{k}\right\}_{k=1}^{\infty} \subset L^{2}\left(\Omega_{T}\right)$ be a sequence converging weakly to $v$ in $L^{2}\left(\Omega_{T}\right)$ with $v \in C_{\bar{u}}$. Further, assume that $\bar{u}_{\nu} \neq 0$.

If $j_{2}^{\prime}\left(\bar{u}_{\nu} ; v_{k}\right) \rightarrow j_{2}^{\prime}\left(\bar{u}_{\nu} ; v\right)$, then the sequence $t \mapsto j_{\Omega}^{\prime}\left(\bar{u}_{\nu}(t) ; v_{k}(t)\right)$ converges weakly in $L^{2}(0, T)$ towards $t \mapsto$ $j_{\Omega}^{\prime}\left(\bar{u}_{\nu}(t) ; v(t)\right)$.

Proof. For $0<t<T$ we set

$$
\Omega_{\bar{u}_{\nu}(t)}^{+}=\left\{x \in \Omega: \bar{u}_{\nu}(x, t)>0\right\}, \quad \Omega_{\bar{u}_{\nu}(t)}^{-}=\left\{x \in \Omega: \bar{u}_{\nu}(x, t)<0\right\},
$$

and

$$
\Omega_{\bar{u}_{\nu}(t)}^{0}=\left\{x \in \Omega: \bar{u}_{\nu}(x, t)=0\right\} .
$$

Then we have for every $w \in L^{2}\left(\Omega_{T}\right)$ by $(3.17)$

$$
j_{\Omega}^{\prime}\left(\bar{u}_{\nu}(t) ; w(t)\right)=\int_{\Omega_{\bar{u}_{\nu}(t)}^{+}} w(x, t) \mathrm{d} x-\int_{\Omega_{\bar{u}_{\nu}(t)}^{-}} w(x, t) \mathrm{d} x+\int_{\Omega_{\bar{u}_{\nu}(t)}^{0}}|w(x, t)| \mathrm{d} x .
$$


Hence, for every $f \in L^{2}(0, T)$ we have

$$
\begin{aligned}
\int_{0}^{T} f(t) j_{\Omega}^{\prime}\left(\bar{u}_{\nu}(t) ; v_{k}(t)\right) \mathrm{d} t= & \int_{0}^{T} f(t) \int_{\Omega_{\bar{u}_{\nu}(t)}^{+}} v_{k}(x, t) \mathrm{d} x \mathrm{~d} t-\int_{0}^{T} f(t) \int_{\Omega_{\bar{u}_{\nu}(t)}^{-}} v_{k}(x, t) \mathrm{d} x \mathrm{~d} t \\
& +\int_{0}^{T} f(t) \int_{\Omega_{\bar{u}_{\nu}(t)}^{0}}\left|v_{k}(x, t)\right| \mathrm{d} x \mathrm{~d} t=I_{k}^{+}+I_{k}^{-}+I_{k}^{0} .
\end{aligned}
$$

The weak convergence $v_{k} \rightarrow v$ in $L^{2}\left(\Omega_{T}\right)$ implies that

$$
\lim _{k \rightarrow \infty} I_{k}^{+}=\int_{0}^{T} f(t) \int_{\Omega_{\bar{u}_{\nu}(t)}^{+}} v(x, t) \mathrm{d} x=I^{+} .
$$

Analogously, we have that $I_{k}^{-} \rightarrow I^{-}$. It remains to prove the convergence $I_{k}^{0} \rightarrow I^{0}$ to conclude the proof. To this end, we observe that the convergence $j_{2}^{\prime}\left(\bar{u}_{\nu} ; v_{k}\right) \rightarrow j_{2}^{\prime}\left(\bar{u}_{\nu} ; v\right)$ means (recall (3.18))

$$
\lim _{k \rightarrow \infty} \int_{0}^{T} \frac{\left\|\bar{u}_{\nu}(t)\right\|_{L^{1}(\Omega)}}{\left\|\bar{u}_{\nu}\right\|_{L^{2}\left(L^{1}\right)}} j_{\Omega}^{\prime}\left(\bar{u}_{\nu}(t) ; v_{k}(t)\right) \mathrm{d} t=\int_{0}^{T} \frac{\left\|\bar{u}_{\nu}(t)\right\|_{L^{1}(\Omega)}}{\left\|\bar{u}_{\nu}\right\|_{L^{2}\left(L^{1}\right)}} j_{\Omega}^{\prime}\left(\bar{u}_{\nu}(t) ; v(t)\right) \mathrm{d} t .
$$

Using again the weak convergence $v_{k} \rightarrow v$, we have

$$
\lim _{k \rightarrow \infty} \int_{0}^{T} \frac{\left\|\bar{u}_{\nu}(t)\right\|_{L^{1}(\Omega)}}{\left\|\bar{u}_{\nu}\right\|_{L^{2}\left(L^{1}\right)}} \int_{\Omega_{\bar{u}_{\nu}(t)}^{+}} v_{k}(x, t) \mathrm{d} x \mathrm{~d} t=\int_{0}^{T} \frac{\left\|\bar{u}_{\nu}(t)\right\|_{L^{1}(\Omega)}}{\left\|\bar{u}_{\nu}\right\|_{L^{2}\left(L^{1}\right)}} \int_{\Omega_{\bar{u}_{\nu}(t)}^{+}} v(x, t) \mathrm{d} x \mathrm{~d} t,
$$

and

$$
\lim _{k \rightarrow \infty} \int_{0}^{T} \frac{\left\|\bar{u}_{\nu}(t)\right\|_{L^{1}(\Omega)}}{\left\|\bar{u}_{\nu}\right\|_{L^{2}\left(L^{1}\right)}} \int_{\Omega_{\bar{u}_{\nu}(t)}^{-}} v_{k}(x, t) \mathrm{d} x \mathrm{~d} t=\int_{0}^{T} \frac{\left\|\bar{u}_{\nu}(t)\right\|_{L^{1}(\Omega)}}{\left\|\bar{u}_{\nu}\right\|_{L^{2}\left(L^{1}\right)}} \int_{\Omega_{\bar{u}_{\nu}(t)}^{-}} v(x, t) \mathrm{d} x \mathrm{~d} t .
$$

The last three limits imply that

$$
\lim _{k \rightarrow \infty} \int_{0}^{T} \frac{\left\|\bar{u}_{\nu}(t)\right\|_{L^{1}(\Omega)}}{\left\|\bar{u}_{\nu}\right\|_{L^{2}\left(L^{1}\right)}} \int_{\Omega_{\bar{u}_{\nu}(t)}^{0}}\left|v_{k}(x, t)\right| \mathrm{d} x \mathrm{~d} t=\int_{0}^{T} \frac{\left\|\bar{u}_{\nu}(t)\right\|_{L^{1}(\Omega)}}{\left\|\bar{u}_{\nu}\right\|_{L^{2}\left(L^{1}\right)}} \int_{\Omega_{\bar{u}_{\nu}(t)}^{0}}|v(x, t)| \mathrm{d} x \mathrm{~d} t .
$$

Since $v \in C_{\bar{u}_{\nu}}$, (4.3) implies that

$$
j_{2}^{\prime}\left(\bar{u}_{\nu} ; v\right)=\int_{\Omega_{T}} \bar{\lambda}_{\nu}(x, t) v(x, t) \mathrm{d} x \mathrm{~d} t .
$$

This equality and (3.10) lead to

$$
\int_{0}^{T} \frac{\left\|\bar{u}_{\nu}(t)\right\|_{L^{1}(\Omega)}}{\left\|\bar{u}_{\nu}\right\|_{L^{2}\left(L^{1}\right)}} \int_{\Omega_{\bar{u}_{\nu}(t)}^{0}}|v(x, t)| \mathrm{d} x \mathrm{~d} t=\int_{0}^{T} \int_{\Omega_{\bar{u}_{\nu}(t)}^{0}} \bar{\lambda}_{\nu}(x, t) v(x, t) \mathrm{d} x \mathrm{~d} t .
$$

Furthermore, the convergence $v_{k} \rightarrow v$ in $L^{2}\left(\Omega_{T}\right)$ implies that

$$
\lim _{k \rightarrow \infty} \int_{\Omega_{T}} \bar{\lambda}_{\nu}(x, t) v_{k}(x, t) \mathrm{d} x \mathrm{~d} t=\int_{\Omega_{T}} \bar{\lambda}_{\nu}(x, t) v(x, t) \mathrm{d} x \mathrm{~d} t .
$$

Therefore, this identity, (5.22) and (5.23) prove that

$$
\lim _{k \rightarrow \infty} \int_{0}^{T} \int_{\Omega_{\bar{u}_{\nu}(t)}^{0}}\left[\frac{\left\|\bar{u}_{\nu}(t)\right\|_{L^{1}(\Omega)}}{\left\|\bar{u}_{\nu}\right\|_{L^{2}\left(L^{1}\right)}}\left|v_{k}(x, t)\right|-\bar{\lambda}_{\nu}(x, t) v_{k}(x, t)\right] \mathrm{d} x \mathrm{~d} t=0 .
$$


Since

$$
\frac{\left\|\bar{u}_{\nu}(t)\right\|_{L^{1}(\Omega)}}{\left\|\bar{u}_{\nu}\right\|_{L^{2}\left(L^{1}\right)}}\left|v_{k}(x, t)\right|-\bar{\lambda}_{\nu}(x, t) v_{k}(x, t) \geq 0 \text { for a.a. }(x, t) \in \Omega_{T}^{0},
$$

with

$$
\Omega_{T}^{0}=\left\{(x, t) \in \Omega_{T}: x \in \Omega_{\bar{u}_{\nu}(t)}^{0}\right\},
$$

we deduce

$$
\frac{\left\|\bar{u}_{\nu}(t)\right\|_{L^{1}(\Omega)}}{\left\|\bar{u}_{\nu}\right\|_{L^{2}\left(L^{1}\right)}}\left|v_{k}(x, t)\right|-\bar{\lambda}_{\nu}(x, t) v_{k}(x, t) \rightarrow 0 \text { strongly in } L^{1}\left(\Omega_{T}^{0}\right) .
$$

Then, we can take a subsequence, denoted in the same way, such that

$$
\frac{\left\|\bar{u}_{\nu}(t)\right\|_{L^{1}(\Omega)}}{\left\|\bar{u}_{\nu}\right\|_{L^{2}\left(L^{1}\right)}}\left|v_{k}(x, t)\right|-\bar{\lambda}_{\nu}(x, t) v_{k}(x, t) \rightarrow 0 \text { for a.a. }(x, t) \in \Omega_{T}^{0} .
$$

Let us prove that

$$
\left|v_{k}(x, t)\right|-\operatorname{sign}\left(\bar{\lambda}_{\nu}(x, t)\right) v_{k}(x, t) \rightarrow 0 \quad \text { for a.a. }(x, t) \in \Omega_{T}^{0} .
$$

According to (3.13), we distinguish three cases for $\bar{\lambda}_{\nu}(x, t)$.

Case I: $\left|\bar{\lambda}_{\nu}(x, t)\right|<\frac{\left\|\bar{u}_{\nu}(t)\right\|_{L^{1}(\Omega)}}{\left\|\bar{u}_{\nu}\right\|_{L^{2}\left(L^{1}\right)}}$. Then, from (5.24) we get

$$
\left(\frac{\left\|\bar{u}_{\nu}(t)\right\|_{L^{1}(\Omega)}}{\left\|\bar{u}_{\nu}\right\|_{L^{2}\left(L^{1}\right)}}-\left|\bar{\lambda}_{\nu}(x, t)\right|\right)\left|v_{k}(x, t)\right| \leq \frac{\left\|\bar{u}_{\nu}(t)\right\|_{L^{1}(\Omega)}}{\left\|\bar{u}_{\nu}\right\|_{L^{2}\left(L^{1}\right)}}\left|v_{k}(x, t)\right|-\bar{\lambda}_{\nu}(x, t) v_{k}(x, t) \rightarrow 0,
$$

hence $\left|v_{k}(x, t)\right| \rightarrow 0$. Then, (5.25) holds in this case.

Case II: $\bar{\lambda}_{\nu}(x, t)=+\frac{\left\|\bar{u}_{\nu}(t)\right\|_{L^{1}(\Omega)}}{\left\|\bar{u}_{\nu}\right\|_{L^{2}\left(L^{1}\right)}}$. In this case, (5.24) implies that $\left|v_{k}(x, t)\right|-v_{k}(x, t) \rightarrow 0$, which coincides with (5.25).

Case III: $\bar{\lambda}_{\nu}(x, t)=-\frac{\left\|\bar{u}_{\nu}(t)\right\|_{L^{1}(\Omega)}}{\left\|\bar{u}_{\nu}\right\|_{L^{2}\left(L^{1}\right)}}$. Now, (5.24) implies that $\left|v_{k}(x, t)\right|+v_{k}(x, t) \rightarrow 0$, which again coincides with (5.25).

Since, the functions $\left|v_{k}(x, t)\right|-\operatorname{sign}\left(\bar{\lambda}_{\nu}(x, t)\right) v_{k}(x, t)$ are bounded in $L^{2}\left(\Omega_{T}^{0}\right)$ independently of $k$, we deduce from (5.25) that $\left|v_{k}(x, t)\right|-\operatorname{sign}\left(\bar{\lambda}_{\nu}(x, t)\right) v_{k}(x, t) \rightarrow 0$ weakly in $L^{2}(\Omega)$. Finally, writing

$$
\left|v_{k}(x, t)\right|=\left[\left|v_{k}(x, t)\right|-\operatorname{sign}\left(\bar{\lambda}_{\nu}(x, t)\right) v_{k}(x, t)\right]+\operatorname{sign}\left(\bar{\lambda}_{\nu}(x, t)\right) v_{k}(x, t),
$$

and using that $\operatorname{sign}\left(\bar{\lambda}_{\nu}(x, t)\right) v_{k}(x, t) \rightarrow \operatorname{sign}\left(\bar{\lambda}_{\nu}(x, t)\right) v(x, t)$ weakly in $L^{2}\left(\Omega_{T}^{0}\right)$, we deduce that $\left|v_{k}\right| \rightarrow \operatorname{sign}\left(\bar{\lambda}_{\nu}\right) v$ weakly in $L^{2}\left(\Omega_{T}^{0}\right)$. Finally, from (5.23) and (3.13), it follows that $|v(x, t)|=\operatorname{sign}\left(\bar{\lambda}_{\nu}\right) v(x, t)$ for a.a. $(x, t) \in \Omega_{T}^{0}$. Therefore, the convergence $\left|v_{k}\right| \rightarrow|v|$ weakly in $L^{2}\left(\Omega_{T}^{0}\right)$ holds, which proves the desired limit

$$
\lim _{k \rightarrow \infty} I_{k}^{0}=\lim _{k \rightarrow \infty} \int_{0}^{T} f(t) \int_{\Omega_{\bar{u}_{\nu}(t)}^{0}}\left|v_{k}(x, t)\right| \mathrm{d} x \mathrm{~d} t=\int_{0}^{T} f(t) \int_{\Omega_{\bar{u}_{\nu}(t)}^{0}}|v(x, t)| \mathrm{d} x \mathrm{~d} t=I^{0} .
$$

The next lemma shows that $j_{2}$ satisfies a second-order Taylor (directional) expansion and is a preparation for Theorem 5.8.

Lemma 5.7. Let $u \in L^{2}\left(\Omega_{T}\right)$ be arbitrary. For any $\delta>0$, there exists $\varepsilon>0$ such that

$$
j_{2}(u+v) \geq j_{2}(u)+j_{2}^{\prime}(u ; v)+\frac{1}{2} j_{2}^{\prime \prime}\left(u ; v^{2}\right)-\frac{\delta}{8}\|v\|_{L^{2}\left(\Omega_{T}\right)}^{2}
$$

holds for all $\|v\|_{L^{2}\left(\Omega_{T}\right)} \leq \varepsilon$. 
Proof. In case $u=0$, the assertion follows from $j_{2}^{\prime}(0 ; v)=j_{2}(v)$ and $j_{2}^{\prime \prime}\left(0 ; v^{2}\right)=0$. Now, let $u \neq 0$. We set

$$
\varepsilon=\min \left\{\frac{\|u\|_{L^{2}\left(L^{1}\right)}}{2|\Omega|}, \frac{\delta\|u\|_{L^{2}\left(L^{1}\right)}^{2}}{32|\Omega|^{3}}\right\} .
$$

First, we observe that

$$
\|u(t)+v(t)\|_{L^{1}(\Omega)} \geq\left|\|u(t)\|_{L^{1}(\Omega)}+j_{\Omega}^{\prime}(u(t) ; v(t))\right| \quad \text { for a.a. } t \in(0, T) .
$$

Let us define $f, g \in L^{2}(0, T)$ by $f(t)=\|u(t)\|_{L^{1}(\Omega)}$ and $g(t)=j_{\Omega}^{\prime}(u(t) ; v(t))$. Then, using the function $\Psi$ defined in Section 3.3, the above estimate yields

$$
j_{2}(u+v)-j_{2}(u) \geq \Psi(f+g)-\Psi(f)=\Psi^{\prime}(f) g+\frac{1}{2} \Psi^{\prime \prime}(f) g^{2}+\frac{1}{6} \Psi^{\prime \prime \prime}\left(f_{\theta}\right) g^{3},
$$

where $f_{\theta}=f+\theta g$, for some $0<\theta<1$. According to (3.26) and applying Hölder's inequality, we get

$$
\left|\Psi^{\prime \prime \prime}\left(f_{\theta}\right) g^{3}\right| \leq \frac{6\|g\|_{L^{2}(0, T)}^{3}}{\left\|f_{\theta}\right\|_{L^{2}(0, T)}^{2}}
$$

From the definition of $g$ and using again the Hölder's inequality, we get

$$
\|g\|_{L^{2}(0, T)} \leq|\Omega|\|v\|_{L^{2}\left(\Omega_{T}\right)}<|\Omega| \varepsilon .
$$

On the other hand, from (5.26) we get

$$
\left\|f_{\theta}\right\|_{L^{2}(0, T)}^{2} \geq\left(\|f\|_{L^{2}(0, T)}-\theta\|g\|_{L^{2}(0, T)}\right)^{2} \geq\left(\|u\|_{L^{2}\left(L^{1}\right)}-|\Omega| \varepsilon\right)^{2} \geq \frac{1}{4}\|u\|_{L^{2}\left(L^{1}\right)}^{2} .
$$

Therefore, using again (5.26), it follows

$$
\left|\Psi^{\prime \prime \prime}\left(f_{\theta}\right) g^{3}\right| \leq \frac{6|\Omega|^{3} \varepsilon}{\frac{1}{4}\|u\|_{L^{2}\left(L^{1}\right)}^{2}}\|v\|_{L^{2}\left(\Omega_{T}\right)}^{2} \leq \frac{3 \delta}{4}\|v\|_{L^{2}\left(\Omega_{T}\right)}^{2} .
$$

Now, from (3.18), (4.6), (3.24) and (3.25), and the definitions of $f$ and $g$, it follows that $\Psi^{\prime}(f) g=j_{2}^{\prime}(u, v)$ and $\Psi^{\prime \prime}(f) g^{2}=j_{2}^{\prime \prime}\left(u ; v^{2}\right)$. Inserting this in (5.27) and using the above estimate for $\Psi^{\prime \prime \prime}\left(f_{\theta}\right) g^{3}$, we obtain the assertion.

Now, we state the theorem on second-order sufficient optimality conditions analogous to Theorem 5.3.

Theorem 5.8. Let $\nu>0$ and assume that $\bar{u}_{\nu}$ satisfies (5.19). Then there exists $\varepsilon>0$ such that

$$
J_{\nu}\left(\bar{u}_{\nu}\right)+\frac{\delta}{4}\left\|u-\bar{u}_{\nu}\right\|_{L^{2}\left(\Omega_{T}\right)}^{2} \leq J_{\nu}(u) \quad \forall u \in \mathbb{K} \cap B_{\varepsilon}\left(\bar{u}_{\nu}\right),
$$

where $B_{\varepsilon}\left(\bar{u}_{\nu}\right)$ denotes the ball of $L^{2}\left(\Omega_{T}\right)$ centered at $\bar{u}_{\nu}$ and with radius $\varepsilon$.

Proof. We take $\varepsilon_{1}$ as in the proof of Theorem 5.3, and $\varepsilon_{2}>0$ such that $\forall u \in \mathbb{K} \cap B_{\varepsilon_{2}}\left(\bar{u}_{\nu}\right)$

$$
\left|\left[F_{\nu}^{\prime \prime}(u)-F_{\nu}^{\prime \prime}\left(\bar{u}_{\nu}\right)\right] v^{2}\right| \leq \frac{\delta}{4 C_{\mathbb{K}}^{2}}\left\|z_{v}\right\|_{L^{2}\left(\Omega_{T}\right)}^{2} \leq \frac{\delta}{4}\|v\|_{L^{2}\left(\Omega_{T}\right)}^{2} \quad \forall v \in L^{2}\left(\Omega_{T}\right),
$$

and $\varepsilon_{3}$ from Lemma 5.7. Then we define

$$
0<\varepsilon \leq \min \left\{\varepsilon_{1}, \varepsilon_{2}, \varepsilon_{3}\right\}
$$


Let us take an arbitrary element $u \in \mathbb{K} \cap B_{\varepsilon}\left(\bar{u}_{\nu}\right)$. If $u-\bar{u}_{\nu} \notin C_{\bar{u}_{\nu}}^{\tau}$, then we can repeat the proof of Theorem 5.3 to obtain (5.29). Let us consider the case $u-\bar{u}_{\nu} \in C_{\bar{u}_{\nu}}^{\tau}$. Making a Taylor expansion of $F_{\nu}(u)$ around $\bar{u}_{\nu}$, using Lemma 5.7 and (5.30), we obtain

$$
\begin{aligned}
J_{\nu}(u)-J_{\nu}\left(\bar{u}_{\nu}\right) \geq & F_{\nu}^{\prime}\left(\bar{u}_{\nu}\right)\left(u-\bar{u}_{\nu}\right)+\mu j_{2}^{\prime}\left(\bar{u}_{\nu} ; u-\bar{u}_{\nu}\right) \\
& +\frac{1}{2} F_{\nu}^{\prime \prime}\left(\bar{u}_{\nu}\right)\left(u-\bar{u}_{\nu}\right)^{2}+\frac{1}{2} j_{2}^{\prime \prime}\left(\bar{u}_{\nu} ;\left(u-\bar{u}_{\nu}\right)^{2}\right) \\
& +\frac{1}{2}\left[F_{\nu}^{\prime \prime}\left(\bar{u}_{\nu}+\theta\left(u-\bar{u}_{\nu}\right)\right)-F_{\nu}^{\prime \prime}\left(\bar{u}_{\nu}\right)\right]\left(u-\bar{u}_{\nu}\right)^{2}-\frac{\delta}{8}\left\|u-\bar{u}_{\nu}\right\|_{L^{2}\left(\Omega_{T}\right)}^{2} \\
\geq & \frac{\delta}{2}\left\|u-\bar{u}_{\nu}\right\|_{L^{2}\left(\Omega_{T}\right)}^{2}-\frac{\delta}{4}\left\|u-\bar{u}_{\nu}\right\|_{L^{2}\left(\Omega_{T}\right)}^{2}=\frac{\delta}{4}\left\|u-\bar{u}_{\nu}\right\|_{L^{2}\left(\Omega_{T}\right)}^{2} .
\end{aligned}
$$

Finally, we analyze the case $\nu=0$. To this end, we need a Taylor expansion of $j_{2}$ similar to Lemma 5.7, but we now have to estimate the remainder in terms of both $j_{2}^{\prime}$ and $j_{2}^{\prime \prime}$, since the second-order condition (5.20) only provides a growth w.r.t. $\left\|z_{v}\right\|_{L^{2}\left(\Omega_{T}\right)}$.

Lemma 5.9. Let $u \in L^{2}\left(\Omega_{T}\right)$ be arbitrary. There exists $\varepsilon>0$ and $C>0$, such that

$$
\left|j_{2}(u+v)-j_{2}(u)-j_{2}^{\prime}(u ; v)-\frac{1}{2} j_{2}^{\prime \prime}\left(u ; v^{2}\right)\right| \leq C\left(\left|j_{2}^{\prime}(u ; v)\right|^{3}+j_{2}^{\prime \prime}\left(u ; v^{2}\right)^{3 / 2}\right)
$$

holds for all $\|v\|_{L^{2}\left(\Omega_{T}\right)} \leq \varepsilon$.

Proof. In case $u=0$, the assertion follows from $j_{2}^{\prime}(0 ; v)=j_{2}(v)$ and $j_{2}^{\prime \prime}\left(0 ; v^{2}\right)=0$. Now, let $u \neq 0$. We set

$$
\varepsilon=\frac{\|u\|_{L^{2}\left(L^{1}\right)}}{2|\Omega|}
$$

We proceed as in the proof of Lemma 5.7, and define $f$ and $g$ in the same way. Thus, (5.27) and (5.28) hold. Moreover, using the lower bound for $\left\|f_{\theta}\right\|_{L^{2}(0, T)}$ proved there, we deduce from (5.28)

$$
\left|\Psi^{\prime \prime \prime}\left(f_{\theta}\right) g^{3}\right| \leq \frac{6}{\frac{1}{4}\|u\|_{L^{2}\left(L^{1}\right)}^{2}}\|g\|_{L^{2}(0, T)}^{3} .
$$

It remains to compare the last terms with the first and second directional derivative of $j_{2}$. To this end we need to compute $\Psi^{\prime \prime}(f) g^{2}$. For convenience, we define

$$
\zeta=\Psi^{\prime}(f) g=\frac{1}{\|f\|_{L^{2}(0, T)}} \int_{0}^{T} f(t) g(t) \mathrm{d} t \quad \text { and } \quad h=g-\frac{\zeta}{\|f\|_{L^{2}(0, T)}} f .
$$

Then, it is easy to check that

$$
\int_{0}^{T} f(t) h(t) \mathrm{d} t=0, \quad\|g\|_{L^{2}(0, T)}^{2}=\|h\|_{L^{2}(0, T)}^{2}+\zeta^{2} \text { and } \Psi^{\prime \prime}(f) g^{2}=\|h\|_{L^{2}(0, T)}^{2} .
$$

Let us recall that $\zeta=\Psi^{\prime}(f) g=j_{2}^{\prime}(u ; v)$. This shows

$$
\begin{aligned}
\left|\Psi^{\prime \prime \prime}\left(f_{\theta}\right) g^{3}\right| \leq \frac{6}{\frac{1}{4}\|u\|_{L^{2}\left(L^{1}\right)}^{2}}\|g\|_{L^{2}(0, T)}^{3} & =C\left(\|h\|_{L^{2}(0, T)}^{2}+\zeta^{2}\right)^{3 / 2} \\
& =C\left(\Psi^{\prime \prime}(f) g^{2}+\left(\Psi^{\prime}(f) g\right)^{2}\right)^{3 / 2} \\
& =C\left(j_{2}^{\prime \prime}\left(u ; v^{2}\right)+j^{\prime}(u ; v)^{2}\right)^{3 / 2} \leq C \sqrt{2}\left(j_{2}^{\prime \prime}\left(u ; v^{2}\right)^{3 / 2}+\left|j^{\prime}(u ; v)\right|^{3}\right)
\end{aligned}
$$

The last inequality follows from the convexity of $z \mapsto z^{3 / 2}$ for $z \geq 0$ and $j_{2}^{\prime \prime}\left(u ; v^{2}\right) \geq 0$. 
Theorem 5.10. Let $\nu=0$ and assume that $\bar{u}_{\nu}$ satisfies (5.20). Then there exists $\varepsilon>0$ such that

$$
J_{\nu}\left(\bar{u}_{\nu}\right)+\frac{\delta}{4}\left\|z_{u-\bar{u}_{\nu}}\right\|_{L^{2}\left(\Omega_{T}\right)}^{2} \leq J_{\nu}(u) \quad \forall u \in \mathbb{K} \cap B_{\varepsilon}\left(\bar{u}_{\nu}\right),
$$

where $z_{u-\bar{u}_{\nu}}=G^{\prime}\left(\bar{u}_{\nu}\right)\left(u-\bar{u}_{\nu}\right)$ and $B_{\varepsilon}\left(\bar{u}_{\nu}\right)$ denotes the ball of $L^{2}\left(\Omega_{T}\right)$ centered at $\bar{u}_{\nu}$ and with radius $\varepsilon$.

Proof. Let us define $\tau^{\prime}=\frac{\tau}{C_{\mathbb{K}}}$ and

$$
E_{\bar{u}_{\nu}}^{\tau^{\prime}}=\left\{v \in \mathcal{T}_{\mathbb{K}}\left(\bar{u}_{\nu}\right): F_{\nu}^{\prime}\left(\bar{u}_{\nu}\right) v+\mu j_{2}^{\prime}\left(\bar{u}_{\nu} ; v\right) \leq \tau^{\prime}\left\|z_{v}\right\|_{L^{2}\left(\Omega_{T}\right)}\right\}
$$

Due to (5.5), we have $\tau^{\prime}\left\|z_{v}\right\|_{L^{2}\left(\Omega_{T}\right)} \leq \tau\|v\|_{L^{2}\left(\Omega_{T}\right)}$, hence $E_{\bar{u}_{\nu}}^{\tau^{\prime}} \subset C_{\bar{u}_{\nu}}^{\tau}$. Therefore, (5.20) holds for all $v \in E_{\bar{u}_{\nu}}^{\tau^{\prime}}$. Then, from (5.14) and (5.20) we get for every $v \in E_{\bar{u}_{\nu}}^{\tau^{\prime}}$ and all $\mu_{0} \in(0, \mu)$

$$
\begin{aligned}
F_{\nu}^{\prime \prime}\left(\bar{u}_{\nu}\right) v^{2}+\mu_{0} j_{2}^{\prime \prime}\left(\bar{u}_{\nu} ; v^{2}\right) & =\left(1-\frac{\mu_{0}}{\mu}\right) F_{\nu}^{\prime \prime}\left(\bar{u}_{\nu}\right) v^{2}+\frac{\mu_{0}}{\mu}\left(F_{\nu}^{\prime \prime}\left(\bar{u}_{\nu}\right) v^{2}+\mu j_{2}^{\prime \prime}\left(\bar{u}_{\nu} ; v^{2}\right)\right) \\
& \geq-\left(1-\frac{\mu_{0}}{\mu}\right) C_{0}\left\|z_{v}\right\|_{L^{2}\left(\Omega_{T}\right)}^{2}+\frac{\mu_{0} \delta}{\mu}\left\|z_{v}\right\|_{L^{2}\left(\Omega_{T}\right)}^{2} .
\end{aligned}
$$

Taking

$$
\frac{3 \delta+4 C_{0}}{4 \delta+4 C_{0}} \mu<\mu_{0}<\mu
$$

we conclude

$$
F_{\nu}^{\prime \prime}\left(\bar{u}_{\nu}\right) v^{2}+\mu_{0} j_{2}^{\prime \prime}\left(\bar{u}_{\nu} ; v^{2}\right) \geq \frac{3 \delta}{4}\left\|z_{v}\right\|_{L^{2}\left(\Omega_{T}\right)}^{2} \forall v \in E_{\bar{u}_{\nu}}^{\tau^{\prime}} .
$$

Given $\varepsilon>0$ to be fixed later, we take an arbitrary element $u \in B_{\varepsilon}\left(\bar{u}_{\nu}\right) \cap \mathbb{K}$ and we distinguish two cases. First, we assume that $u-\bar{u}_{\nu} \notin E_{\bar{u}_{\nu}}^{\tau^{\prime}}$. Then, we argue similarly to the proof of Theorem 5.4 and use (5.5) and (5.14)

$$
\begin{aligned}
J_{\nu}(u)-J_{\nu}\left(\bar{u}_{\nu}\right) & \geq F_{\nu}^{\prime}\left(\bar{u}_{\nu}\right)\left(u-\bar{u}_{\nu}\right)+\mu j_{2}^{\prime}\left(\bar{u}_{\nu} ; u-\bar{u}_{\nu}\right)+\frac{1}{2} F_{\nu}^{\prime \prime}\left(\bar{u}_{\nu}+\theta\left(u-\bar{u}_{\nu}\right)\right)\left(u-\bar{u}_{\nu}\right)^{2} \\
& \geq \tau^{\prime}\left\|z_{u-\bar{u}_{\nu}}\right\|_{L^{2}\left(\Omega_{T}\right)}-\frac{1}{2} C_{0}\left\|z_{u-\bar{u}_{\nu}}\right\|_{L^{2}\left(\Omega_{T}\right)}^{2} \\
& \geq \frac{\tau^{\prime}}{\varepsilon}\left\|u-\bar{u}_{\nu}\right\|_{L^{2}\left(\Omega_{T}\right)}\left\|z_{u-\bar{u}_{\nu}}\right\|_{L^{2}\left(\Omega_{T}\right)}-\frac{1}{2} C_{0}\left\|z_{u-\bar{u}_{\nu}}\right\|_{L^{2}\left(\Omega_{T}\right)}^{2} \\
& \geq\left(\frac{\tau^{\prime}}{\varepsilon C_{\mathbb{K}}}-\frac{1}{2} C_{0}\right)\left\|z_{u-\bar{u}_{\nu}}\right\|_{L^{2}\left(\Omega_{T}\right)}^{2} \\
& =\left(\frac{\tau}{\varepsilon C_{\mathbb{K}}^{2}}-\frac{1}{2} C_{0}\right)\left\|z_{u-\bar{u}_{\nu}}\right\|_{L^{2}\left(\Omega_{T}\right)}^{2} \geq \frac{\delta}{4}\left\|z_{u-\bar{u}_{\nu}}\right\|_{L^{2}\left(\Omega_{T}\right)}^{2}
\end{aligned}
$$

assuming that $0<\varepsilon \leq \varepsilon_{1}$ with $\varepsilon_{1}$ chosen as in the proof of Theorem 5.4.

Now, we suppose that $u-\bar{u}_{\nu} \in E_{\bar{u}_{\nu}}^{\tau^{\prime}}$. By using Lemma 5.9, we obtain the Taylor expansion

$$
\begin{aligned}
J_{\nu}(u)-J_{\nu}\left(\bar{u}_{\nu}\right) \geq F_{\nu}^{\prime} & \left(\bar{u}_{\nu}\right)\left(u-\bar{u}_{\nu}\right)+\mu j_{2}^{\prime}\left(\bar{u}_{\nu} ; u-\bar{u}_{\nu}\right) \\
& +\frac{1}{2} F_{\nu}^{\prime \prime}\left(\bar{u}_{\nu}\right)\left(u-\bar{u}_{\nu}\right)^{2}+\frac{\mu_{0}}{2} j_{2}^{\prime \prime}\left(\bar{u}_{\nu} ;\left(u-\bar{u}_{\nu}\right)^{2}\right) \\
& +\frac{1}{2}\left(F_{\nu}^{\prime \prime}\left(\bar{u}_{\nu}+\theta\left(u-\bar{u}_{\nu}\right)\right)-F_{\nu}^{\prime \prime}\left(\bar{u}_{\nu}\right)\right)\left(u-\bar{u}_{\nu}\right)^{2} \\
& -C\left(\left|j_{2}^{\prime}\left(\bar{u}_{\nu} ; u-\bar{u}_{\nu}\right)\right|^{3}+j_{2}^{\prime \prime}\left(\bar{u}_{\nu} ;\left(u-\bar{u}_{\nu}\right)^{2}\right)^{3 / 2}\right) \\
& +\frac{\mu-\mu_{0}}{2} j_{2}^{\prime \prime}\left(\bar{u}_{\nu} ;\left(u-\bar{u}_{\nu}\right)^{2}\right) .
\end{aligned}
$$


The first line on the right-hand side is non-negative, since $\bar{u}_{\nu}$ satisfies the first-order condition (3.4). For the second line, we use (5.34). The third line is bounded by using (5.7) which holds with $\rho=\delta / 4$ for $\varepsilon$ small enough. In the last two lines, we use

$$
C j_{2}^{\prime \prime}\left(\bar{u}_{\nu} ;\left(u-\bar{u}_{\nu}\right)^{2}\right)^{3 / 2} \leq \frac{\mu-\mu_{0}}{2} j_{2}^{\prime \prime}\left(\bar{u}_{\nu} ;\left(u-\bar{u}_{\nu}\right)^{2}\right),
$$

which again holds for $\varepsilon$ small enough. Now, the above inequality simplifies to

$$
J_{\nu}(u)-J_{\nu}\left(\bar{u}_{\nu}\right) \geq \frac{3 \delta}{4}\left\|z_{v}\right\|_{L^{2}\left(\Omega_{T}\right)}^{2}-\frac{\delta}{4}\left\|z_{v}\right\|_{L^{2}\left(\Omega_{T}\right)}^{2}-C\left|j_{2}^{\prime}\left(\bar{u}_{\nu} ; u-\bar{u}_{\nu}\right)\right|^{3} .
$$

It remains to bound $j_{2}^{\prime}\left(\bar{u}_{\nu}, u-\bar{u}_{\nu}\right)$ by $\left\|z_{v}\right\|_{L^{2}\left(\Omega_{T}\right)}$. Since $u-\bar{u}_{\nu} \in E_{\bar{u}_{\nu}}^{\tau^{\prime}}$, we have

$$
0 \leq F_{\nu}^{\prime}\left(\bar{u}_{\nu}\right)\left(u-\bar{u}_{\nu}\right)+\mu j_{2}^{\prime}\left(\bar{u}_{\nu} ; u-\bar{u}_{\nu}\right) \leq \tau^{\prime}\left\|z_{u-\bar{u}_{\nu}}\right\|_{L^{2}\left(\Omega_{T}\right)} .
$$

This yields

$$
\mu\left|j_{2}^{\prime}\left(\bar{u}_{\nu}, u-\bar{u}_{\nu}\right)\right| \leq \tau^{\prime}\left\|z_{u-\bar{u}_{\nu}}\right\|_{L^{2}\left(\Omega_{T}\right)}+\left|F_{\nu}^{\prime}\left(\bar{u}_{\nu}\right)\left(u-\bar{u}_{\nu}\right)\right| .
$$

Now, from (2.9) we obtain

$$
F_{\nu}^{\prime}\left(\bar{u}_{\nu}\right)\left(u-\bar{u}_{\nu}\right)=\int_{\Omega_{T}} \frac{\partial L}{\partial y}\left(x, t, \bar{y}_{\nu}(x, t)\right) z_{u-\bar{u}_{\nu}} \mathrm{d} x \mathrm{~d} t .
$$

Then, it is enough to use (2.5) with $M=\left\|\bar{y}_{\nu}\right\|_{L^{\infty}\left(\Omega_{T}\right)}$ to deduce

$$
\left|F_{\nu}^{\prime}\left(\bar{u}_{\nu}\right)\left(u-\bar{u}_{\nu}\right)\right| \leq\left\|\psi_{M}\right\|_{L^{2}\left(\Omega_{T}\right)}\left\|z_{u-\bar{u}_{\nu}}\right\|_{L^{2}\left(\Omega_{T}\right)} .
$$

Using this estimate in (5.35), (5.36) we find

$$
\begin{aligned}
J_{\nu}(u)+J_{\nu}\left(\bar{u}_{\nu}\right) & \geq \frac{\delta}{2}\left\|z_{v}\right\|_{L^{2}\left(\Omega_{T}\right)}^{2}-C\left|j_{2}^{\prime}\left(\bar{u}_{\nu} ; u-\bar{u}_{\nu}\right)\right|^{3} \\
& \geq \frac{\delta}{2}\left\|z_{v}\right\|_{L^{2}\left(\Omega_{T}\right)}^{2}-C\left\|z_{v}\right\|_{L^{2}\left(\Omega_{T}\right)}^{3} \\
& \geq \frac{\delta}{4}\left\|z_{v}\right\|_{L^{2}\left(\Omega_{T}\right)}^{2}
\end{aligned}
$$

for $\varepsilon$ small enough.

\subsection{Problem $\left(\mathrm{P}_{\nu}^{3}\right)$}

For the problem $\left(\mathrm{P}_{\nu}^{3}\right)$ we have that $J_{\nu}^{\prime \prime}\left(\bar{u}_{\nu} ; v^{2}\right)=F_{\nu}^{\prime \prime}\left(\bar{u}_{\nu}\right) v^{2}+\mu j_{3}^{\prime \prime}\left(\bar{u}_{\nu} ; v^{2}\right)$, where $j_{3}^{\prime \prime}\left(\bar{u}_{\nu} ; v^{2}\right)$ is given by $(4.7)$. Analogously to Theorems 5.1 and 5.5 we have the next result.

Theorem 5.11. Let us assume that $\nu>0$. Then the following statements are equivalent

$$
\begin{aligned}
& J_{\nu}^{\prime \prime}\left(\bar{u}_{\nu} ; v^{2}\right)>0 \forall v \in C_{\bar{u}_{\nu}} \backslash\{0\}, \\
& \exists \delta>0 \text { and } \tau>0 \text { such that } J_{\nu}^{\prime \prime}\left(\bar{u}_{\nu} ; v^{2}\right) \geq \delta\|v\|_{L^{2}\left(\Omega_{T}\right)}^{2} \forall v \in C_{\bar{u}_{\nu}}^{\tau}, \\
& \exists \delta>0 \text { and } \tau>0 \text { such that } J_{\nu}^{\prime \prime}\left(\bar{u}_{\nu} ; v^{2}\right) \geq \delta\left\|z_{v}\right\|_{L^{2}\left(\Omega_{T}\right)}^{2} \forall v \in C_{\bar{u}_{\nu}}^{\tau} .
\end{aligned}
$$

Proof. It is enough to prove that (5.37) implies (5.38). The proof follows the same steps of that of Theorem 5.5. The only difference is the way of obtaining the inequality

$$
J_{\nu}^{\prime \prime}\left(\bar{u}_{\nu} ; v^{2}\right) \leq \liminf _{k \rightarrow \infty} J_{\nu}^{\prime \prime}\left(\bar{u}_{\nu} ; v_{k}^{2}\right) \leq 0 .
$$

To prove this it is enough to observe that the mapping $L^{2}\left(\Omega_{T}\right) \ni v \mapsto j_{3}^{\prime \prime}\left(\bar{u}_{\nu} ; v^{2}\right) \in \mathbb{R}$ is convex and lower semicontinuous. 
Now, we prove the theorem analogous to Theorems 5.3 and 5.8.

Theorem 5.12. Let $\nu>0$ and assume that $\bar{u}_{\nu}$ satisfies (5.37). Then there exist $\varepsilon>0$ and $\delta^{\prime}>0$ such that

$$
J_{\nu}\left(\bar{u}_{\nu}\right)+\frac{\delta^{\prime}}{2}\left\|u-\bar{u}_{\nu}\right\|_{L^{2}\left(\Omega_{T}\right)}^{2} \leq J_{\nu}(u) \quad \forall u \in \mathbb{K} \cap B_{\varepsilon}\left(\bar{u}_{\nu}\right)
$$

where $B_{\varepsilon}\left(\bar{u}_{\nu}\right)$ denotes the ball of $\left.L^{\infty}\left(\Omega ; L^{2}(0, T)\right)\right)$ centered at $\bar{u}_{\nu}$ and with radius $\varepsilon$, i.e.

$$
B_{\varepsilon}\left(\bar{u}_{\nu}\right)=\left\{u \in L^{\infty}\left(\Omega ; L^{2}(0, T)\right): \operatorname{ess}_{\sup } \in \Omega\left\|u(x)-\bar{u}_{\nu}(x)\right\|_{L^{2}(0, T)}<\varepsilon\right\} .
$$

Proof. We assume that $\bar{u}_{\nu} \not \equiv 0$, the case $\bar{u}_{\nu} \equiv 0$ being immediate. We argue by contradiction. If (5.40) does not hold for any $\varepsilon>0$ and $\delta^{\prime}>0$, then for any integer $k \geq 1$ there exists an element $u_{k} \in \mathbb{K}$ such that

$$
\left\|u_{k}-\bar{u}_{\nu}\right\|_{L^{\infty}\left(\Omega ; L^{2}(0, T)\right)}<\frac{1}{k} \text { and } J_{\nu}\left(u_{k}\right)<J_{\nu}\left(\bar{u}_{\nu}\right)+\frac{1}{2 k}\left\|u_{k}-\bar{u}_{\nu}\right\|_{L^{2}\left(\Omega_{T}\right)}^{2}
$$

Let us define

$$
\rho_{k}=\left\|u_{k}-\bar{u}_{\nu}\right\|_{L^{2}\left(\Omega_{T}\right)}<\frac{\sqrt{|\Omega|}}{k} \text { and } v_{k}=\frac{1}{\rho_{k}}\left(u_{k}-\bar{u}_{\nu}\right) .
$$

We take a subsequence, if necessary, such that $v_{k} \rightarrow v$ in $L^{2}\left(\Omega_{T}\right)$. The proof is split into three steps.

Step I. $v \in C_{\bar{u}_{\nu}}$. First we observe that $v_{k} \in \mathcal{T}_{\mathbb{K}}\left(\bar{u}_{\nu}\right)$ for every $k$. Since $\mathcal{T}_{\mathbb{K}}\left(\bar{u}_{\nu}\right)$ is convex and closed in $L^{2}\left(\Omega_{T}\right)$, we have that $v \in \mathcal{T}_{\mathbb{K}}\left(\bar{u}_{\nu}\right)$ as well. On the other hand, since $j_{3}$ is a Lipschitz and convex function we have that

$$
\begin{aligned}
j_{3}^{\prime}\left(\bar{u}_{\nu} ; v\right) & \leq \liminf _{k \rightarrow \infty} j_{3}^{\prime}\left(\bar{u}_{\nu} ; v_{k}\right) \leq \liminf _{k \rightarrow \infty} \frac{j_{3}\left(\bar{u}_{\nu}+\rho_{k} v_{k}\right)-j_{3}\left(\bar{u}_{\nu}\right)}{\rho_{k}} \\
& =\liminf _{k \rightarrow \infty} \frac{j_{3}\left(u_{k}\right)-j_{3}\left(\bar{u}_{\nu}\right)}{\rho_{k}} .
\end{aligned}
$$

The last equality is an immediate consequence of the definition of $v_{k}$ in (5.42). Using this inequality and (5.41) we get

$$
\begin{aligned}
F_{\nu}^{\prime}\left(\bar{u}_{\nu}\right) v+\mu j_{3}^{\prime}\left(\bar{u}_{\nu} ; v\right) & \leq \liminf _{k \rightarrow \infty} \frac{1}{\rho_{k}}\left\{\left[F_{\nu}\left(\bar{u}_{\nu}+\rho_{k} v_{k}\right)-F_{\nu}\left(\bar{u}_{\nu}\right)\right]+\mu\left[j_{3}\left(\bar{u}_{\nu}+\rho_{k} v_{k}\right)-j_{3}\left(\bar{u}_{\nu}\right)\right]\right\} \\
& =\liminf _{k \rightarrow \infty} \frac{1}{\rho_{k}}\left[J_{\nu}\left(u_{k}\right)-J_{\nu}\left(\bar{u}_{\nu}\right)\right] \\
& \leq \liminf _{k \rightarrow \infty} \frac{1}{2 k \rho_{k}}\left\|u_{k}-\bar{u}_{\nu}\right\|_{L^{2}\left(\Omega_{T}\right)}^{2}=\liminf _{k \rightarrow \infty} \frac{\rho_{k}}{2 k}=0 .
\end{aligned}
$$

This inequality and (4.2) imply that $F_{\nu}^{\prime}\left(\bar{u}_{\nu}\right) v+\mu j_{3}^{\prime}\left(\bar{u}_{\nu} ; v\right)=0$, hence $v \in C_{\bar{u}_{\nu}}$.

Step II. $v=0$. For $\sigma>0$ small we define

$$
\Omega_{\sigma}=\left\{x \in \Omega:\left\|\bar{u}_{\nu}(x)\right\|_{L^{2}(0, T)} \geq \sigma\right\} \text { and } j_{3, \sigma}(u)=\int_{\Omega_{\sigma}}\|u(x)\|_{L^{2}(0, T)} \mathrm{d} x .
$$


For $f, g \in L^{2}(0, T)$ defined by $f=\bar{u}_{\nu}(x)$ and $g=v_{k}(x)$, making a Taylor expansion of $\Phi\left(f+\rho_{k} g\right)$ around $f$, and using (3.24)-(3.26) we deduce

$$
\begin{aligned}
j_{3, \sigma}\left(\bar{u}_{\nu}+\rho_{k} v_{k}\right)-j_{3, \sigma}\left(\bar{u}_{\nu}\right)= & \rho_{k} \int_{\Omega_{\sigma}} \frac{1}{\left\|\bar{u}_{\nu}(x)\right\|_{L^{2}(0, T)}} \int_{0}^{T} \bar{u}_{\nu}(x, t) v_{k}(x, t) \mathrm{d} t \mathrm{~d} x \\
& +\frac{\rho_{k}^{2}}{2} \int_{\Omega_{\sigma}} \frac{1}{\left\|\bar{u}_{\nu}(x)\right\|_{L^{2}(0, T)}}\left\{\int_{0}^{T} v_{k}^{2}(x, t) \mathrm{d} t-\left(\int_{0}^{T} \frac{\bar{u}_{\nu}(x, t)}{\left\|\bar{u}_{\nu}(x)\right\|_{L^{2}(0, T)}} v_{k}(x, t) \mathrm{d} t\right)^{2}\right\} \mathrm{d} x \\
& +\frac{\rho_{k}^{3}}{6} \int_{\Omega_{\sigma}} \frac{3}{\left\|u_{\vartheta_{k}}(x)\right\|_{L^{2}(0, T)}^{3}}\left\{\frac{1}{\left\|u_{\vartheta_{k}}(x)\right\|_{L^{2}(0, T)}^{2}}\left(\int_{0}^{T} u_{\vartheta_{k}}(x, t) v_{k}(x, t) \mathrm{d} t\right)^{3}\right. \\
& \left.-\left(\int_{0}^{T} v_{k}(x, t)^{2} \mathrm{~d} t\right)\left(\int_{0}^{T} u_{\vartheta_{k}}(x, t) v_{k}(x, t) \mathrm{d} t\right)\right\} \mathrm{d} x \\
= & \rho_{k} j_{3, \sigma}^{\prime}\left(\bar{u}_{\nu} ; v_{k}\right)+\frac{\rho_{k}^{2}}{2} j_{3, \sigma}^{\prime \prime}\left(\bar{u}_{\nu} ; v_{k}^{2}\right)+\frac{\rho_{k}^{3}}{6} j_{3, \sigma}^{\prime \prime \prime}\left(u_{\vartheta_{k}} ; v_{k}^{3}\right),
\end{aligned}
$$

where $u_{\vartheta_{k}}=\bar{u}_{\nu}+\vartheta_{k} \rho_{k} v_{k}$ with $0 \leq \vartheta_{k}(x) \leq 1$. Observe that relations (5.42) and (5.41) lead to

$$
\left\|u_{\vartheta_{k}}(x)\right\|_{L^{2}(0, T)} \geq \sigma-\vartheta_{k} \rho_{k}\left\|v_{k}(x)\right\|_{L^{2}(0, T)} \geq \sigma-\vartheta_{k}\left\|u_{k}-\bar{u}_{\nu}\right\|_{L^{\infty}\left(\Omega ; L^{2}(0, T)\right.} \geq \sigma-\frac{1}{k} \geq \frac{\sigma}{2}>0
$$

for all $k \geq \frac{2}{\sigma}$. Hence, the above integrals are finite for every $k \geq \frac{2}{\sigma}$.

Now, taking into account that $\Phi^{\prime \prime}(f) g^{2} \geq 0$ for every $f, g \in L^{2}(0, T)$, we get

$$
\begin{aligned}
j_{3}\left(\bar{u}_{\nu}+\rho_{k} v_{k}\right)-j_{3}\left(\bar{u}_{\nu}\right)= & \rho_{k} \int_{\Omega_{\bar{u}_{\nu}}^{0}}\left\|v_{k}(x)\right\|_{L^{2}(0, T)} \\
& +\int_{\Omega_{\bar{u}_{\nu}} \backslash \Omega_{\sigma}}\left\{\left\|\left(\bar{u}_{\nu}+\rho_{k} v_{k}\right)(x)\right\|_{L^{2}(0, T)}-\left\|\bar{u}_{\nu}(x)\right\|_{L^{2}(0, T)}\right\} \mathrm{d} x \\
& +\left[j_{3, \sigma}\left(\bar{u}_{\nu}+\rho_{k} v_{k}\right)-j_{3, \sigma}\left(\bar{u}_{\nu}\right)\right] \\
\geq & \rho_{k} j_{3}^{\prime}\left(\bar{u}_{\nu} ; v_{k}\right)+\frac{\rho_{k}^{2}}{2} j_{3, \sigma}^{\prime \prime}\left(\bar{u}_{\nu} ; v_{k}^{2}\right)+\frac{\rho_{k}^{3}}{6} j_{3, \sigma}^{\prime \prime \prime}\left(u_{\vartheta_{k}} ; v_{k}^{3}\right) .
\end{aligned}
$$

From (5.41) we get

$$
\begin{aligned}
\frac{\rho_{k}^{2}}{2 k}>J_{\nu}\left(\bar{u}_{\nu}+\rho_{k} v_{k}\right)-J_{\nu}\left(\bar{u}_{\nu}\right) \geq & \rho_{k}\left\{F_{\nu}^{\prime}\left(\bar{u}_{\nu}\right) v_{k}+\mu j_{3}^{\prime}\left(\bar{u}_{\nu} ; v_{k}\right)\right\} \\
& +\frac{\rho_{k}^{2}}{2}\left\{F_{\nu}^{\prime \prime}\left(\bar{u}_{\nu}\right) v_{k}^{2}+\mu j_{3, \sigma}^{\prime \prime}\left(\bar{u}_{\nu} ; v_{k}^{2}\right)\right\} \\
& +\frac{\rho_{k}^{2}}{2}\left[F_{\nu}^{\prime \prime}\left(u_{\theta_{k}}\right)-F_{\nu}^{\prime \prime}\left(\bar{u}_{\nu}\right)\right] v_{k}^{2}+\mu \frac{\rho_{k}^{3}}{6} j_{3, \sigma}^{\prime \prime \prime}\left(u_{\vartheta_{k}} ; v_{k}^{3}\right),
\end{aligned}
$$

where $u_{\theta_{k}}=\bar{u}_{\nu}+\theta_{k} \rho_{k}\left(u_{k}-\bar{u}_{\nu}\right)$ with $0 \leq \theta_{k} \leq 1$. Since $v_{k} \in \mathcal{T}_{\mathbb{K}}\left(\bar{u}_{\nu}\right)$, we deduce from (4.2)

$$
\frac{\rho_{k}^{2}}{2 k}>\frac{\rho_{k}^{2}}{2}\left\{F_{\nu}^{\prime \prime}\left(\bar{u}_{\nu}\right) v_{k}^{2}+\mu j_{3, \sigma}^{\prime \prime}\left(\bar{u}_{\nu} ; v_{k}^{2}\right)\right\}+\frac{\rho_{k}^{2}}{2}\left[F_{\nu}^{\prime \prime}\left(u_{\theta_{k}}\right)-F_{\nu}^{\prime \prime}\left(\bar{u}_{\nu}\right)\right] v_{k}^{2}+\mu \frac{\rho_{k}^{3}}{6} j_{3, \sigma}^{\prime \prime \prime}\left(u_{\vartheta_{k}} ; v_{k}^{3}\right) .
$$

Dividing this expression by $\rho_{k}^{2} / 2$ we obtain

$$
F_{\nu}^{\prime \prime}\left(\bar{u}_{\nu}\right) v_{k}^{2}+\mu j_{3, \sigma}^{\prime \prime}\left(\bar{u}_{\nu} ; v_{k}^{2}\right)<\left|\left[F_{\nu}^{\prime \prime}\left(u_{\theta_{k}}\right)-F_{\nu}^{\prime \prime}\left(\bar{u}_{\nu}\right)\right] v_{k}^{2}\right|+\mu \frac{\rho_{k}}{3}\left|j_{3, \sigma}^{\prime \prime \prime}\left(u_{\vartheta_{k}} ; v_{k}^{3}\right)\right| .
$$


From Lemma 5.2 and the identity $\left\|v_{k}\right\|_{L^{2}\left(\Omega_{T}\right)}=1$ we deduce

$$
\lim _{k \rightarrow \infty}\left|\left[F_{\nu}^{\prime \prime}\left(u_{\theta_{k}}\right)-F_{\nu}^{\prime \prime}\left(\bar{u}_{\nu}\right)\right] v_{k}^{2}\right|=0 .
$$

Let us estimate the last term of (5.43). By using Hölder's inequality and the expression of $j_{3, \sigma}^{\prime \prime \prime}\left(u_{\vartheta_{k}} ; v_{k}^{3}\right)$ we obtain

$$
\left|j_{3, \sigma}^{\prime \prime \prime}\left(u_{\vartheta_{k}} ; v_{k}^{3}\right)\right| \leq 6 \int_{\Omega_{\sigma}} \frac{\left\|v_{k}(x)\right\|_{L^{2}(0, T)}^{3}}{\left\|u_{\vartheta_{k}}(x)\right\|_{L^{2}(0, T)}^{2}} \mathrm{~d} x .
$$

Using that $\left\|u_{\vartheta_{k}}(x)\right\|_{L^{2}(0, T)} \geq \frac{\sigma}{2}$ for every $k$ large enough and $\left\|v_{k}\right\|_{L^{2}\left(\Omega_{T}\right)}=1$ we get

$$
\mu \frac{\rho_{k}}{3}\left|j_{3, \sigma}^{\prime \prime \prime}\left(u_{\vartheta_{k}} ; v_{k}^{3}\right)\right| \leq \frac{8 \mu}{k \sigma^{2}} \int_{\Omega_{\sigma}}\left\|v_{k}(x)\right\|_{L^{2}(0, T)}^{2} \mathrm{~d} x \rightarrow 0 \text { as } k \rightarrow \infty .
$$

Now, from (5.43), (5.44) and (5.45) the following inequality follows

$$
F_{\nu}^{\prime \prime}\left(\bar{u}_{\nu}\right) v^{2}+\mu j_{3, \sigma}^{\prime \prime}\left(\bar{u}_{\nu} ; v^{2}\right) \leq \liminf _{k \rightarrow \infty}\left\{F_{\nu}^{\prime \prime}\left(\bar{u}_{\nu}\right) v_{k}^{2}+\mu j_{3, \sigma}^{\prime \prime}\left(\bar{u}_{\nu} ; v_{k}^{2}\right)\right\} \leq 0 \quad \forall \sigma>0 .
$$

Hence, taking the limit as $\sigma \rightarrow 0$ we conclude

$$
F_{\nu}^{\prime \prime}\left(\bar{u}_{\nu}\right) v^{2}+\mu j_{3}^{\prime \prime}\left(\bar{u}_{\nu} ; v^{2}\right) \leq 0 .
$$

According to (5.37), this is possible only if $v=0$.

Step III. Contradiction. Since $v=0$, then $z_{v_{k}} \rightarrow 0$ strongly in $L^{2}\left(\Omega_{T}\right)$. Then, from the expression of $F_{\nu}^{\prime \prime}$ given by (2.10), the fact that $j_{3, \sigma}^{\prime \prime}\left(\bar{u}_{\nu} ; v_{k}^{2}\right) \geq 0$ and the identity $\left\|v_{k}\right\|_{L^{2}\left(\Omega_{T}\right)}=1$, we deduce from (5.43), (5.44) and (5.45)

$$
\nu \leq \liminf _{k \rightarrow \infty}\left\{F_{\nu}^{\prime \prime}\left(\bar{u}_{\nu}\right) v_{k}^{2}+\mu j_{3, \sigma}^{\prime \prime}\left(\bar{u}_{\nu} ; v_{k}^{2}\right)\right\} \leq 0,
$$

which contradict the assumption $\nu>0$.

In the above proof, the fact that $\nu>0$ was crucial to get the contradiction. The proof of the sufficient second order conditions for the case $\nu=0$ is an open problem for us. An important difference between the cases $j_{2}$ and $j_{3}$ is that there is no singularity in $j_{2}^{\prime \prime}\left(u ; v^{2}\right)$ if $u \neq 0$, however we can have singularities in the integral defining $j_{3}^{\prime \prime}\left(u ; v^{2}\right)$ for $u \neq 0$ when $\|u(x)\|_{L^{2}(0, T)} \rightarrow 0$. The integrals in (4.7) can be $+\infty$. This renders the handling of the remainder terms in the Taylor expansions of $j_{3}(u)$ around $\bar{u}_{\nu}$ rather complicated. To be more precise, we were not able to show a remainder term estimate parallel to Lemma 5.9 for $j_{3}$. This estimate, however, was crucial in the proof of Theorem 5.10 since in the case of $\nu=0,(5.39)$ only provides a growth in terms of $\left\|z_{v}\right\|_{L^{2}\left(\Omega_{T}\right)}^{2}$ and not in terms of $\|v\|_{L^{2}\left(\Omega_{T}\right)}^{2}$

\section{REFERENCES}

[1] J.F. Bonnans and A. Shapiro, Perturbation analysis of optimization problems. Springer Series in Operations Research. Springer-Verlag, New York (2000).

[2] E. Casas, Pontryagin's principle for state-constrained boundary control problems of semilinear parabolic equations. SIAM J. Control Optim. 35 (1997) 1297-1327.

[3] E. Casas, Second order analysis for bang-bang control problems of PDEs. SIAM J. Control Optim. 50 (2012) $2355-2372$.

[4] E. Casas and K. Kunisch, Optimal Control of Semilinear Elliptic Equations in Measure Spaces. SIAM J. Control Optim. 52 (2014) 339-364.

[5] E. Casas, R. Herzog, and G. Wachsmuth, Optimality conditions and error analysis of semilinear elliptic control problems with $L^{1}$ cost functional. SIAM J. Optim. 22 (2012) 795-820.

[6] E. Casas, C. Clason, and K. Kunisch, Parabolic control problems in measure spaces with sparse solutions. SIAM J. Control Optim. 51 (2013) 28-63.

[7] J. Dunn, On second order sufficient optimality conditions for structured nonlinear programs in infinite-dimensional function spaces, in Mathematical Programming with Data Perturbations, edited by A. Fiacco. Marcel Dekker (1998) 83-107. 
[8] R.E. Edwards, Functional analysis. Theory and applications, Corrected reprint of the 1965 original. Dover Publications Inc., New York (1995).

[9] I. Ekeland and R. Temam, Convex Analysis and Variational Problems. In vol. 28 of Classics in Applied Mathematics. SIAM, Philadelphia (1999).

[10] R. Herzog, J. Obermeier, and G. Wachsmuth, Annular and sectorial sparsity in optimal control of elliptic equations. Comput. Optim. Appl. 62 (2015) 157-180.

[11] R. Herzog, G. Stadler, and G. Wachsmuth, Directional sparsity in optimal control of partial differential equations. SIAM J. Control Optim. 50 (2012) 943-963.

[12] A.D. Ioffe and V.M. Tichomirov, Theorie der Extremalaufgaben. VEB Deutscher Verlag der Wissenschaften, Berlin (1979).

[13] K. Kunisch, K. Pieper, and B. Vexler, Measure valued directional sparsity for parabolic optimal control problems. SIAM J. Control Optim. 52 (2014) 3078-3108.

[14] J. Nečas, Les Méthodes Directes en Théorie des Equations Elliptiques. Editeurs Academia, Prague (1967).

[15] G. Stadler, Elliptic optimal control problems with $L^{1}$-control cost and applications for the placement of control devices. Comput. Optim. Appl. 44 (2009) 159-181. 